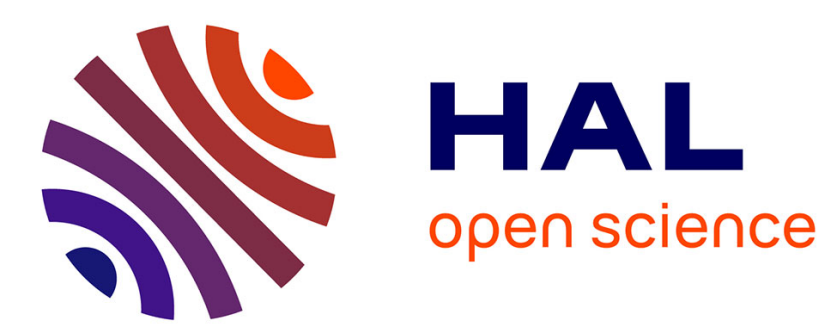

\title{
Réalisations de Hodge des motifs de Voevodsky
}

Florence Lecomte, Nathalie Wach

\section{To cite this version:}

Florence Lecomte, Nathalie Wach. Réalisations de Hodge des motifs de Voevodsky. Manuscripta mathematica, 2013, 141, pp.663-697. 10.1007/s00229-012-0587-5 . hal-00437302v3

\section{HAL Id: hal-00437302 \\ https://hal.science/hal-00437302v3}

Submitted on 20 Feb 2012

HAL is a multi-disciplinary open access archive for the deposit and dissemination of scientific research documents, whether they are published or not. The documents may come from teaching and research institutions in France or abroad, or from public or private research centers.
L'archive ouverte pluridisciplinaire HAL, est destinée au dépôt et à la diffusion de documents scientifiques de niveau recherche, publiés ou non, émanant des établissements d'enseignement et de recherche français ou étrangers, des laboratoires publics ou privés. 


\title{
REALISATION DE HODGE DES MOTIFS DE VOEVODSKY
}

\author{
Florence LECOMTE et Nathalie WACH \\ IRMA - Strasbourg
}

\begin{abstract}
RESUME
Pour un sous-corps du corps des complexes, nous définissons un foncteur de la catégorie des motifs géométriques de Voevodsky vers la catégorie des $\mathbf{Z}$-complexes de Hodge mixtes de Deligne [D74]. Les filtrations par le poids et de Hodge sont représentées par des foncteurs de troncature d'un complexe des poids à la Bondarko [Bo10] pour la première et du complexe de De Rham [LW09] pour l'autre.
\end{abstract}

\section{Introduction}

Après l'invention des motifs par Grothendieck, Deligne a défini les structures de Hodge mixtes comme de très bonnes approximations de structures motiviques : elles forment des catégories abéliennes et tensorielles et sont construites à partir de structures élémentaires dites pures. Il est donc essentiel de construire les réalisations de Hodge des motifs de Voevodsky, ce qu'a fait rationnellement Annette Huber en [H00] et [H04]. Dans cet article, qui fait suite à nos travaux sur les réalisations de De Rham [LW09] et Betti [L08] nous construisons une réalisation de Hodge à partir de foncteurs représentables.

Soit $k$ un sous-corps du corps des nombres complexes. En [L08], l'une des auteurs a construit pour tout plongement $\sigma: k \hookrightarrow \mathbf{C}$ un foncteur de réalisation topologique $t_{\sigma}$ de la catégorie des complexes motiviques de Voevodsky $\mathbf{D M}^{-}(k)$ dans la catégorie dérivée $D(A b)$ des groupes abéliens et défini le foncteur de réalisation de Betti

$$
\mathbf{H}_{\sigma}(\mathbf{M}, q)=\operatorname{Hom}_{D(A b)}\left(t_{\sigma}(\mathbf{M}),(2 i \pi)^{q} \mathbf{Z}\right) .
$$

En [LW09] nous avons construit un ind-motif de De Rham pour représenter le foncteur de réalisation de De Rham dans la catégorie des complexes motiviques non bornés de Cisinski-Déglise $\mathbf{D M}(k)$ (cf $[\mathrm{CD} 09])$

$$
\mathbf{H}_{D R}(\mathbf{M})=\operatorname{Hom}_{\mathbf{D M}(k)}(\mathbf{M}, \boldsymbol{\Omega}) .
$$

Notons que ces réalisations sont définies comme cohomologies de complexes, à savoir

$$
\mathbf{H}_{\sigma}^{p}(\mathbf{M}, q)=H^{p}\left(\mathrm{R} \cdot \operatorname{Hom}_{D(A b)}\left(t_{\sigma}(\mathbf{M}),(2 i \pi)^{q} \mathbf{Z}\right)\right) \text { et } \mathbf{H}_{D R}^{p}(\mathbf{M})=H^{p}\left(\mathrm{R} \cdot \operatorname{Hom}_{\mathbf{D M}(k)}(\mathbf{M}, \boldsymbol{\Omega})\right) .
$$

Lorsque l'on se restreint à la catégorie des motifs géométriques $\mathbf{D M}_{\mathrm{gm}}(k)$, le théorème de De Rham se généralise en un théorème de comparaison

$$
\mathbf{H}_{D R}(\mathbf{M}) \otimes \mathbf{C} \simeq \mathbf{H}_{\sigma}(\mathbf{M}, q) \otimes \mathbf{C}
$$

dont les flèches sont également définies au niveau des complexes.

Bondarko [Bo10] a muni la catégorie $\mathbf{D M}_{\mathrm{gm}}(k)$ d'une structure de poids qui permet de définir sur les foncteurs de réalisations des filtrations par le poids $W$ compatibles à l'isomorphisme de comparaison.

Pour tout motif géométrique $\mathbf{M}$, choisissons un complexe des poids $\mathbf{M}^{(\cdot)}$. Alors le complexe différentiel gradué

$$
\oplus_{i \in \mathbf{Z}} \mathrm{R} \cdot \operatorname{Hom}_{\mathbf{D M}(k)}\left(\mathbf{M}^{(i)}, \mathbf{\Omega}\right)
$$

qui s'envoie dans le complexe $\mathrm{R} \cdot \operatorname{Hom}_{\mathbf{D M}(k)}(\mathbf{M}, \boldsymbol{\Omega})$, calcule la réalisation de De Rham de $\mathbf{M}$ et la filtration par le poids est définie par le fonteur de troncature à droite sur la première variable du complexe de motifs $\mathbf{M}^{(\cdot)}$

$$
\left.W_{n} \mathrm{R} \cdot \operatorname{Hom}_{\mathbf{D M}(k)}(\mathbf{M}, \boldsymbol{\Omega})=\operatorname{Im}\left(\oplus_{i \leq n} \mathrm{R} \cdot \operatorname{Hom}_{\mathbf{D M}(k)}\left(\mathbf{M}^{(i)}, \boldsymbol{\Omega}\right)\right) \rightarrow \mathrm{R} \cdot \operatorname{Hom}_{\mathbf{D M}(k)}(\mathbf{M}, \boldsymbol{\Omega})\right) .
$$

On a de même, avec les notations évidentes

$W_{n} \mathrm{R} \cdot \operatorname{Hom}_{D(A b)}\left(t_{\sigma}(\mathbf{M}),(2 i \pi)^{q} \mathbf{Z}\right)=\operatorname{Im}\left(\oplus_{i \leq n} \mathrm{R} \cdot \operatorname{Hom}_{D(A b)}\left(t_{\sigma}\left(\mathbf{M}^{(i)}\right),(2 i \pi)^{q}\right) \rightarrow \mathrm{R} \cdot \operatorname{Hom}_{D(A b)}\left(t_{\sigma}(\mathbf{M}),(2 i \pi)^{q} \mathbf{Z}\right)\right)$.

Sur $\mathrm{R} \operatorname{Hom}\left(\mathbf{M}^{(\cdot)}, \boldsymbol{\Omega}\right)$ on tronque la deuxième variable en considérant la filtration bête de $\boldsymbol{\Omega}$ pour définir une filtration $F$, dite filtration de Hodge. Celle-ci s'étend à $\mathrm{R} \cdot \operatorname{Hom}\left(\mathbf{M}^{(\cdot)}, \boldsymbol{\Omega}\right) \otimes \mathbf{C}$ où elle induit une filtration opposée $\bar{F}$. Notre principal résultat est 
Theoreme 0.1 Pour tout motif géométrique $\mathbf{M}$ décomposé en un complexe des poids $\mathbf{M}^{(\cdot)}$, les données

$$
\left(\mathrm{R} \cdot \operatorname{Hom}_{D(A b)}\left(t_{\sigma}(\mathbf{M}),(2 i \pi)^{q} \mathbf{Z}\right), \mathrm{R} \cdot \operatorname{Hom}(\mathbf{M}, \boldsymbol{\Omega}), \mathrm{R} \cdot \operatorname{Hom}(\mathbf{M}, \boldsymbol{\Omega}) \otimes \mathbf{C}\right)
$$

munies des filtrations induites par les images des foncteurs de troncatures sur $\mathbf{M}^{(\cdot)}$ et $\boldsymbol{\Omega}$ définissent un foncteur vers la catégorie des $\mathbf{Z}$-complexes de Hodge mixtes au sens de Deligne [D74].

Le théorème signifie, entre autres, que les cohomologies des complexes $\mathrm{R}$ · Hom ne dépendent pas du choix du complexe des poids et que la filtration de Hodge est bien définie, bien que, a priori, en tronquant bêtement $\boldsymbol{\Omega}$ on perde l'invariance d'homotopie et on sorte de la catégorie des complexes motiviques.

Comme chez Deligne [D74] il est nécessaire de définir les réalisations au niveau des complexes $\mathrm{R}$ Hom, afin d'obtenir une filtration de Hodge sur $\mathbf{H}_{D R}(\mathbf{M})$ et non sur son gradué par les poids. Néanmoins, les démonstrations consistant à vérifier des propriétés cohomologiques, les résultats seront énoncés en termes de foncteurs cohomologiques.

Bien qu'en [L08] et [LW09], les foncteurs de réalisations aient été définis sur la catégorie $\mathbf{D M}^{-}(k)$ des complexes motiviques de Voevodsky, pour le théorème de comparaison ou la dégénérescence des suites spectrales associées aux filtrations, nous avons besoin de résultats de finitude et devons donc nous restreindre à la catégorie des motifs géométriques $\mathbf{D M}_{\mathrm{gm}}(k)$ introduite par Voevodsky. Dans la catégorie $\mathbf{D M}^{-}(k)$, la catégorie $\mathbf{D M}_{\mathrm{gm}}(k)$ est la sous-catégorie pleine additive épaisse engendrée par les motifs $\mathbf{M}(X)$ des schémas projectifs lisses [MVW 14.1]. Cela signifie que la catégorie $\mathbf{D M}_{\mathrm{gm}}(k)$ est construite à partir des sommes finies de motifs de schémas projectifs lisses avec les deux propriétés suivantes :

- les facteurs directs des motifs géométriques sont géométriques;

- pour tout triangle distingué $A \rightarrow B \rightarrow C \rightarrow A[1]$, si deux des trois motifs $A, B$ et $C$ sont géométriques, le troisième l'est aussi.

PRINCIPE 0.2.

Soit $H: \mathbf{D M}^{-, \text {eff }}(k) \rightarrow \mathcal{A}$ un foncteur cohomologique vers une catégorie abélienne $\mathcal{A}$. Soit $\mathcal{B}$ une sous-catégorie pleine de $\mathcal{A}$, abélienne, stable par facteur direct et extension. Si $H(\mathbf{M}(X)[n])$ est un objet de $\mathcal{B}$ pour tout schéma $X$ lisse et projectif sur $k$ et tout entier $n$, alors $H$ induit un foncteur

$$
H: \mathbf{D M}_{\mathrm{gm}}^{\mathrm{eff}}(k) \rightarrow \mathcal{B}
$$

Si de plus la catégorie $\mathcal{B}$ est tensorielle, si $H$ est multiplicatif et $H(\mathbf{Z}(1))$ est inversible dans $\mathcal{B}$, alors $H$ induit un foncteur

$$
H: \mathbf{D M}_{\mathrm{gm}}(k) \rightarrow \mathcal{B}
$$

Dans cet énoncé, l'hypothèse $\mathcal{A}$ abélienne est nécessaire pour considérer un foncteur cohomologique.

Nous commençons par rappeler les résultats de Voevodsky et Bondarko. Puis nous revoyons brièvement la construction du motif de De Rham et définissons les filtrations par le poids et de Hodge. Ensuite nous construisons la réalisation de Betti qui a été résumée en [L08]. Elle est équivalente à celle construite par Ayoub en théorie homotopique des schémas [A10]. Finalement nous comparons ces deux réalisations pour construire la réalisation de Hodge, qui nous permet d'étendre aux motifs géométriques les cohomologies de Deligne-Beilinson. Nous terminons en montrant que rationnellement nos réalisations coïncident avec celles précédemment construites par Huber [H00].

Remerciements : ce travail a bénéficié de discussions avec J. Wildeshaus sur les travaux de Bondarko et avec A. Huber sur la filtration de Hodge. Qu'ils en soient remerciés.

Conventions Par la suite tous les corps sont supposés de caractéristique 0 et les schémas sont séparés de type fini sur un corps. On note $\operatorname{Sm}(k)$ la catégorie des schémas lisses sur $k$, dont les morphismes sont les morphismes de schémas. 


\section{Motifs de Voevodsky}

La catégorie motivique dans laquelle nous travaillons est la catégorie triangulée $\mathbf{D} \mathbf{M}^{-}(k)$ de Voevodsky ([V-TCM] ) dont nous appelons les objets complexes motiviques. Cette catégorie est obtenue par une série de localisations à partir de la catégorie des complexes de faisceaux sur le site Smcor $(k)$ des correspondances finies et topologie de Nisnevich.

\subsection{Rappels sur les sites et topos}

Rappelons quelques notions utiles de [SGA4]. Un site est une catégorie munie d'une topologie de Grothendieck [SGA4 II 1.15]. Pour un site $\mathcal{C}$, on note $\widehat{\mathcal{C}}$ (resp. $\widetilde{\mathcal{C}}$ ) la catégorie des préfaisceaux (resp. faisceaux) d'ensembles du site $\mathcal{C}$. La catégorie $\widetilde{\mathcal{C}}$ est appelée topos associé au site $\mathcal{C}$. Les catégories $\widehat{\mathcal{C}}$ et $\widetilde{\mathcal{C}}$ sont munies de foncteurs canoniques $\mathcal{C} \rightarrow \widehat{\mathcal{C}}$ et $\mathcal{C} \rightarrow \widetilde{\mathcal{C}}$ qui à un objet $C$ de $\mathcal{C}$ associe respectivement le préfaisceau et le faisceau représentés par l'objet $C$. Si $\mathcal{C}$ et $\mathcal{C}^{\prime}$ sont deux sites, un foncteur $u: \mathcal{C} \rightarrow \mathcal{C}^{\prime}$ entre les catégories sous-jacentes induit par composition un foncteur $\hat{u}^{*}: \widehat{\mathcal{C}}^{\prime} \rightarrow \widehat{\mathcal{C}}$. Ce foncteur $\hat{u}^{*}$ admet un adjoint à gauche $u !: \widehat{\mathcal{C}} \rightarrow \widehat{\mathcal{C}}^{\prime}$ qui prolonge le foncteur d'origine $u$ dans le sens que le diagramme suivant commute

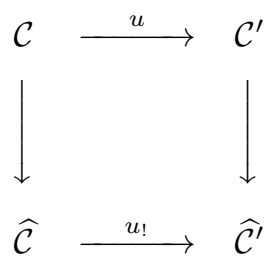

où les flèches verticales sont les foncteurs canoniques.

Le foncteur de prolongement $u$ ! est défini de la façon suivante : pour tout objet $C^{\prime}$ de $\mathcal{C}^{\prime}$ on note $I_{u}^{C^{\prime}}$ la catégorie des couples $(S, f)$ où $S$ est un objet de $\mathcal{C}$ et $f$ un morphisme $f: C^{\prime} \rightarrow u(S)$ dans $\mathcal{C}^{\prime}$ [loc. cit. I 5] et $\left(I_{u}^{C^{\prime}}\right)^{\text {op }}$ la catégorie opposée. On pose

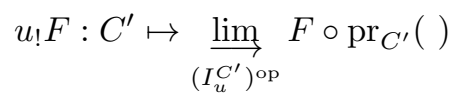

où $\operatorname{pr}_{C^{\prime}}$ est le foncteur de $I_{u}^{C^{\prime}}$ vers $\mathcal{C}$ qui au couple $(S, f)$ associe l'objet $S$.

Si de plus, le foncteur $u$ est continu, c'est-à-dire que pour tout faisceau $G$ sur $\mathcal{C}^{\prime}$ le préfaisceau $C \mapsto G \circ u(C)$ est un faisceau sur $\mathcal{C}$, alors le foncteur $u^{*}$ induit un foncteur $u_{s}: \widetilde{\mathcal{C}}^{\prime} \rightarrow \widetilde{\mathcal{C}}$ et ce foncteur $u_{s}$ admet un adjoint à gauche $u^{s}$ qui prolonge $u$

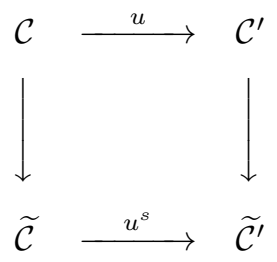

Le foncteur $u^{s}$ est le composé du foncteur de prolongement $u$ ! défini plus haut avec le foncteur faisceau associé. Par construction, il est exact à droite et commute aux limites inductives. S'il est de plus exact à gauche, il est foncteur image inverse $u^{s}=\Phi^{*}$ d'un morphisme de topos $\Phi: \widetilde{\mathcal{C}}^{\prime} \rightarrow \widetilde{\mathcal{C}}$ (loc. cit. IV.3.1.). Suivant toujours [SGA4], on note $\widetilde{\mathcal{C}}_{A b}$ le topos abélien associé au site $\mathcal{C}$, c'est-à-dire la catégorie des faisceaux en groupes abéliens sur $\mathcal{C}$.

Rappelons qu'un foncteur d'une catégorie triangulée $\mathcal{T}$ vers une catégorie abélienne $\mathcal{A}$ est dit cohomologique [Ver77] s'il transforme tout triangle distingué en suite exacte. En particulier, pour un foncteur cohomologique $H$ et $p$ un entier, nous notons $H^{p}$ le foncteur de $\mathcal{T}$ vers $\mathcal{A}$ défini par $H^{p}(M)=H(M[-p])$; ceci permet d'associer à tout triangle distingué une suite exacte longue. Les foncteurs Hom sont des foncteurs cohomologiques. 


\subsection{Les correspondances finies}

Le groupe $\operatorname{Cor}(X, Y)$ des correspondances finies entre deux schémas lisses $X$ et $Y$ est le groupe abélien libre engendré par les sous-variétés fermées irréductibles de $X \times_{\operatorname{Spec}(k)} Y$ qui sont finies et surjectives sur une composante irréductible de $X$. Cette définition reste valable pour un schéma $Y$ quelconque.

Les correspondances finies se comportent mieux que les cycles classiques : il existe des morphismes image inverse et image directe pour tous les morphismes entre schémas lisses et elles se composent comme les correspondances de Grothendieck [Ma68]. Elles permettent de définir la catégorie Smcor $(k)$ des correspondances finies, dont les objets sont les schémas lisses sur $k$ et les morphismes, les correspondances finies. La catégorie $\operatorname{Smcor}(k)$ est additive, pour l'union disjointe, et tensorielle, pour le produit fibré sur Spec $k$. Le foncteur canonique $\operatorname{Sm}(k) \rightarrow \operatorname{Smcor}(k)$ qui envoie tout morphisme sur son graphe est compatible à ces structures. On appelle préfaisceau avec transferts un foncteur contravariant de $\operatorname{Smcor}(k)$ vers la catégorie $A b$ des groupes abéliens; un faisceau de Nisnevich avec transferts est un préfaisceau avec transferts qui est un faisceau pour la topologie de Nisnevich, la topologie totalement décomposée de [N89], intermédiaire entre la topologie de Zariski et la topologie étale.

La topologie de Nisnevich munit les catégories $\operatorname{Sm}(k)$ et Smcor(k) ([BV08]4.3.) de topologie de Grothendieck. Le fait que Smcor $(k)$ soit un site pour la topologie de Nisnevich provient de ce que l'image inverse d'un point (anneau hensélien) par une correspondance finie est un point. Suivant Voevodsky, on préférera noter $\operatorname{Shv}_{N i s}(\operatorname{Smcor}(k))$ et $\operatorname{Shv}_{e ́ t}(\operatorname{Smcor}(k))$ les topos abéliens des correspondances finies. Le foncteur canonique $\operatorname{Sm}(k) \rightarrow \operatorname{Smcor}(k)$ est continu (un préfaisceau sur Smcor $(k)$ est un faisceau si c'est un faisceau sur $\operatorname{Sm}(k))$ et cocontinu car les cribles $\operatorname{sur} \operatorname{Sm}(k)$ et $\operatorname{Smcor}(k)$ sont les mêmes.

\subsection{Les catégories motiviques}

1.3.1. Faisceaux de Nisnevich avec transferts

Les catégories motiviques sont construites à partir de la catégorie $\operatorname{Shv}_{N i s}(\operatorname{Smcor}(k))$ des faisceaux de Nisnevich avec transferts. Le faisceau $\mathbf{Z}_{t r}(X)$ (noté $L(X)$ dans $[\mathrm{V}-\mathrm{TCM}]$ ) est le faisceau de Nisnevich représenté par le schéma $X$ sur Smcor $(k)$ : pour tout schéma lisse $U$, on a $\mathbf{Z}_{t r}(X)(U)=$ $\operatorname{Cor}(U, X)$. Notons que le faisceau $\mathbf{Z}_{t r}(X)$ est défini pour $\mathrm{X}$ quelconque. Le produit des schémas permet de définir le produit tensoriel des faisceaux avec transferts

$$
\mathbf{Z}_{t r}(X) \otimes \mathbf{Z}_{t r}(Y)=\mathbf{Z}_{t r}\left(X \times_{\operatorname{Spec}(k)} Y\right)
$$

pour toute paire de schémas lisses $(X, Y)$. Soulignons une propriété de la topologie de Nisnevich ([V-TCM] Prop 3.1.3) :

Proposition 1.3.1.1. (Voevodsky [V-TCM]) Soit $X$ un schéma lisse sur $k$ et $\mathcal{U}=\left\{U_{i} \rightarrow X\right\}$ un recouvrement de Nisnevich de $X$. Notons $U$ l'union disjointe $U=\amalg U_{i}$ et $\check{N}(\mathcal{U} / X)$ le complexe de faisceaux

$$
\cdots \rightarrow \mathbf{Z}_{t r}\left(U \times_{X} U\right) \rightarrow \mathbf{Z}_{t r}(U) \rightarrow \mathbf{Z}_{t r}(X) \rightarrow 0
$$

avec les différentielles égales à la somme alternée des morphismes induits par les projections. Alors le complexe $\check{N}(\mathcal{U} / X)$ est acyclique pour la topologie de Nisnevich.

Comme tout schéma lisse de type fini sur un corps peut être recouvert par une famille de schémas lisses quasi-projectifs, cette proposition permet de résoudre les faisceaux $\mathbf{Z}_{t r}(X)$, pour $X$ schéma lisse de type fini par un complexe formé de sommes $\coprod_{\alpha} \mathbf{Z}_{t r}\left(X_{\alpha}\right)$ où les schémas $\left(X_{\alpha}\right)$ sont quasiprojectifs lisses. C'est pourquoi dans nos constructions nous pourrons supposer que les schémas sont quasi-projectifs.

La proposition (1.3.1.1.) reste valable en topologie étale mais pas en topologie de Zariski (loc. cit.). 1.3.2. $\mathbf{A}^{1}$ - localisation.

La catégorie $\mathbf{D M}^{-, \text {eff }}(k)$ des complexes motiviques effectifs est la localisation de la catégorie dérivée $D^{-}\left(\operatorname{Shv}_{N i s}(\operatorname{Smcor}(k))\right.$ des complexes, bornés supérieurement, de faisceaux de Nisnevich avec transferts par la sous-catégorie épaisse engendrée par les complexes du type $\mathbf{Z}_{t r}\left(X \times_{\operatorname{Spec}(k)} \mathbf{A}_{k}^{1}\right) \rightarrow \mathbf{Z}_{t r}(X)$. On appelle $\mathbf{A}_{k}^{1}$-équivalence tout morphisme de $D^{-}\left(\operatorname{Shv}_{N i s}(\operatorname{Smcor}(k))\right)$ qui induit un isomorphisme sur $\mathbf{D} \mathbf{M}^{-}(k)$. On note $\mathbf{M}(X)$ le complexe motivique associé au schéma 
lisse $X$ : il est représenté dans $\mathbf{D M}^{- \text {,eff }}(k)$ par le complexe singulier simplicial $C_{*}\left(\mathbf{Z}_{t r}(X)\right)$ associé à $X$, aussi appelé complexe de Suslin du schéma $X$. Pour un faisceau $F$, le complexe $C_{*}(F)$ est le complexe de faisceaux défini par

$$
C_{n}(F)(X)=F\left(X \times \Delta^{n}\right)
$$

où $\Delta^{\prime}$ est le schéma cosimplicial standard $\Delta^{n}=\operatorname{Spec} k\left[z_{0}, \ldots, z_{n}\right] /\left(\sum_{0 \leq i \leq n} z_{i}-1\right)$ et la différentielle est induite par la somme alternée des morphismes de coface.

Le produit sur $\operatorname{Smcor}(k)$ se transporte sur $\mathbf{D} \mathbf{M}^{-, \text {eff }}(k)$ et on a pour toute paire $(X, Y)$ de schémas lisses

$$
\mathbf{M}(X) \otimes \mathbf{M}(Y)=\mathbf{M}\left(X \times_{\operatorname{Spec}(k)} Y\right) .
$$

Le motif $\mathbf{M}\left(\mathbf{P}^{1}\right)$ de la droite projective se scinde en $\mathbf{M}\left(\mathbf{P}^{1}\right)=\mathbf{Z} \oplus \mathbf{Z}(1)[2]$ où $\mathbf{Z}=\mathbf{M}(\operatorname{Spec} k)$ est le motif du point et $\mathbf{Z}(1)$ est le motif de Tate, motif réduit de $\mathbf{G}_{m}$. La catégorie $\mathbf{D M}^{-}(k)$ des complexes motiviques est obtenue à partir de la catégorie $\mathbf{D} \mathbf{M}^{- \text {eff }}(k)$ en inversant le motif de Tate. Le théorème de simplification ("cancellation theorem") de Voevodsky (cf [MVW] 16.25) permet d'identifier la catégorie des complexes motiviques effectifs à une sous-catégorie pleine de $\mathbf{D M}^{-}(k)$. Pour tout entier $q$ et tout complexe motivique $\mathbf{M}$ on note $\mathbf{M}(q)$ le produit $\mathbf{M} \otimes \mathbf{Z}(q)$.

REMARQUES : hors le théorème de simplification, ces constructions sont formelles et restent valables en topologie étale sur $\operatorname{Smcor}(k)$ mais aussi sur $\operatorname{Smcor}(\mathbf{C})$ pour la topologie analytique. Le théorème de simplification est trivial en topologie analytique : le motif de Tate analytique est le complexe concentré en degré $0, \mathbf{Z}(q)_{a n}=(2 i \pi)^{q}$ (cf ci-dessous, proposition 3.1.7.).

Outre la catégorie $\mathbf{D} \mathbf{M}_{e ́ t}^{-}(k)$ (loc. cit.) on peut construire la catégorie triangulée $\mathbf{D} \mathbf{M}_{a n}^{-}$à partir de la catégorie $\operatorname{Smcor}(\mathbf{C})$ et de la topologie analytique. Elle est équipée d'un foncteur induit par le changement de topologie

$$
t_{a n}: \mathbf{D M}^{-}(\mathbf{C}) \rightarrow \mathbf{D M}_{a n}^{-}
$$

Spécifique à la topologie de Nisnevich est le théorème d'invariance d'homotopie qui permet d'identifier les objets $\mathbf{A}^{1}$-locaux.

1.3.3. Complexes $\mathbf{A}^{1}$-locaux en topologie de Nisnevich

On dit qu'un (pré)-faisceau $F$ est invariant par homotopie si pour tout schéma lisse $X$, la projection $X \times_{\operatorname{Spec}(k)} \mathbf{A}_{k}^{1} \rightarrow X$ induit un isomorphisme $F(X) \simeq F\left(X \times_{\operatorname{Spec}(k)} \mathbf{A}_{k}^{1}\right)$. Un résulat fondamental de Voevodsky est le théorème d'invariance d'homotopie :

Theoreme 1.3.3.1. (Voevodsky [V-TCM] 3.1.12) Soit F un faisceau de Nisnevich invariant par homotopie. Alors le faisceau de cohomologie associé est également invariant par homotopie et on a pour tout schéma $X$ lisse et tout entier $i$ des isomorphismes

$$
\begin{aligned}
& H_{Z a r}^{i}(X, F) \simeq H_{Z a r}^{i}\left(X \times_{\operatorname{Spec}(k)} \mathbf{A}_{k}^{1}, F\right) \\
& H_{N i s}^{i}(X, F) \simeq H_{N i s}^{i}\left(X \times_{\operatorname{Spec}(k)} \mathbf{A}_{k}^{1}, F\right) .
\end{aligned}
$$

Le théorème d'invariance d'homotopie (1.3.3.1.) permet d'identifier $\mathbf{D M}^{- \text {,eff }}(k)$ à une souscatégorie pleine de la catégorie dérivée $D^{-}\left(\operatorname{Shv}_{N i s}(\operatorname{Smcor}(k))\right)$ : en effet, $\mathbf{D M}^{- \text {,eff }}(k)$ en est la souscatégorie formée des complexes, bornés supérieurement, de faisceaux de Nisnevich avec transferts, qui sont à cohomologie invariante par homotopie.

Par la suite nous considérons des complexes de faisceaux avec transferts $L$ qui sont $\mathbf{A}^{1}$-locaux, c'est-à-dire tels que pour tout complexe motivique on a

$$
\operatorname{Hom}_{D^{-}\left(\operatorname{Shv}_{N i s}(\operatorname{Smcor}(k))\right)}\left(\mathbf{M}, L^{\cdot}\right)=\operatorname{Hom}_{\mathbf{D M}^{-,}, \operatorname{eff}}(k)\left(\mathbf{M}, L^{\cdot}\right) \text {. }
$$

En travaillant dans la catégorie $D^{-}\left(\operatorname{Shv}_{N i s}(\operatorname{Smcor}(k))\right)$ nous nous ramenons à dériver des foncteurs de la catégorie abélienne des faisceaux de Nisnevich avec transferts. Cette catégorie a assez d'injectifs [MVW 6.19] et les foncteurs Ext sont les dérivés des foncteurs Hom. Plus précisément, nous notons $\mathrm{R}$ : Hom le bifoncteur dérivé

$$
D^{-}\left(\operatorname{Shv}_{N i s}(\operatorname{Smcor}(k))\right) \times D^{+}\left(\operatorname{Shv}_{N i s}(\operatorname{Smcor}(k))\right) \rightarrow D(\mathcal{A} b)
$$


défini par R $\operatorname{Hom}(M, N)=\operatorname{Hom}^{\prime}(M, I)$ où $M$ (resp. $\left.N\right)$ est un complexe de faisceaux de Nisnevich avec transferts borné supérieurement (resp. inférieurement), $I$ est une résolution injective de $N$ et $\operatorname{Hom}(-,-)$ est le complexe $n \mapsto \operatorname{Hom}(-,-[n])$. Le foncteur Ext est le foncteur cohomologique associé et on a pour tout $M$ de $D^{-}\left(\operatorname{Shv}_{N i s} \operatorname{Smcor} k\right)$ et tout complexe borné $N$

$$
\operatorname{Ext}^{i}(M, N) \simeq H^{i}(\mathrm{R} \cdot \operatorname{Hom}(M, N)) \simeq \operatorname{Hom}_{D^{-}\left(\operatorname{Shv}_{N i s}(\operatorname{Smcor} k)\right)}(M, N[i]) .
$$

Nous utilisons abondamment le résultat suivant :

Theoreme 1.3.3.2. (Voevodsky [V-TCM]) Si $L$ est un complexe borné $\mathbf{A}^{1}$-local de $\mathbf{D} \mathbf{M}^{-, \text {eff }}(k)$, alors pour tout motif $\mathbf{M}(X)$ d'un schéma $X$ lisse sur $k$, on a des isomorphismes

$$
\begin{aligned}
\operatorname{Hom}_{\mathbf{D M}^{-}(k)}\left(\mathbf{M}(X), L^{\cdot}[i]\right) & \simeq \operatorname{Hom}_{D^{-}\left(\operatorname{Shv}_{N i s}(\operatorname{Smcor}(k))\right)}(\mathbf{M}(X), L \cdot[i]) \simeq \mathbb{H}_{N i s}^{i}\left(X, L^{\cdot}\right) \\
& \simeq \operatorname{Hom}_{D^{-}\left(\operatorname{Shv}_{\text {Zar }}(\operatorname{Smcor}(k))\right)}(\mathbf{M}(X), L \cdot[i]) \simeq \mathbb{H}_{Z a r}^{i}\left(X, L^{\cdot}\right)
\end{aligned}
$$

où $\mathbb{H}_{N i s}^{i}$ (resp. $\mathbb{H}_{Z a r}^{i}$ ) désigne l'hypercohomologie de Nisnevich (resp. Zariski) des complexes de faisceaux.

Le lemme ci-dessous permet de passer de la catégorie $D^{-}\left(\operatorname{Shv}_{N i s}(\operatorname{Smcor}(k))\right)$ à $\mathbf{D M}^{-, \text {eff }}(k)$.

Lemme d'homotopie 1.3.3.2. Soit F : $D^{-}\left(\operatorname{Shv}_{N i s}(\operatorname{Smcor}(k))\right) \rightarrow A\left(\operatorname{resp} . F: D^{-}\left(\operatorname{Shv}_{N i s}(\operatorname{Smcor}(k))\right) \rightarrow\right.$ $T$ ) un foncteur cohomologique dans une catégorie abélienne $A$ (resp. foncteur exact dans une catégorie triangulée $T$ ) qui vérifie la propriété suivante :

pour tout schéma $X$ lisse sur $k$, la première projection $\pi_{X}: X \times_{k} \mathbf{A}^{1} \rightarrow X$ induit un isomorphisme

$$
F\left(\mathbf{Z}_{t r}(X)\right) \simeq F\left(\mathbf{Z}_{t r}\left(X \times_{k} \mathbf{A}^{1}\right)\right) .
$$

Alors $F$ se factorise en un foncteur cohomologique $F: \mathbf{D M}^{-, \text {eff }}(k) \rightarrow A$ (resp. foncteur exact $\left.F: \mathbf{D M}^{-, \text {eff }}(k) \rightarrow T\right)$.

Demonstration : Il suffit de montrer que si $f: K \rightarrow K^{\prime}$ est une $\mathbf{A}^{1}$-équivalence de complexes de faisceaux avec transferts, alors l'image $F(C f)$ du cône de $f$ est nulle. Par définition, le cône $C f$ est dans la plus petite catégorie épaisse contenant le cône $C \pi_{X}$ de $\pi_{X}$ et stable par somme directe. Etant cohomologique (resp. exact), le foncteur $F$ commute aux sommes finies et facteurs directs et on se ramène à montrer que l'image $F\left(C \pi_{X}\right)$ est nulle, ce qu'implique l'hypothèse.

\subsubsection{Motifs géométriques}

La catégorie $\mathbf{D} \mathbf{M}_{\mathrm{gm}}^{\mathrm{eff}}(k)$ des motifs géométriques effectifs est la sous-catégorie épaisse de $\mathbf{D} \mathbf{M}^{-, \text {eff }}(k)$ engendrée par les motifs $\mathbf{M}(X)$ des schémas lisses. Comme le corps $k$ vérifie la résolution des singularités, la catégorie $\mathbf{D} \mathbf{M}_{\mathrm{gm}}^{\text {eff }}(k)$ est engendrée par les motifs des schémas projectifs et lisses et elle contient les motifs de tous les schémas sur $k$. Cette catégorie est également construite par double localisation (invariance d'homotopie et Mayer-Vietoris) de la catégorie homotopique des complexes bornés de $\operatorname{Smcor}(k)$. C'est cette deuxième construction qu'utilise Bondarko [Bo09] pour munir $\mathbf{D M}_{\mathrm{gm}}^{\mathrm{eff}}(k)$ d'une structure différentielle graduée. La catégorie des motifs géométriques $\mathbf{D M}_{\mathrm{gm}}(k)$ est obtenue par inversion du motif de Tate.

Dans la catégorie des motifs géométriques $\mathbf{D M}_{\mathrm{gm}}(k)$, Voevodsky définit un Hom interne $\underline{\text { Hom }}$ et une dualité $\mathbf{M}^{*}=\underline{\operatorname{Hom}}(\mathbf{M}, \mathbf{Z})$. Il associe également à tout schéma $X$ un motif à support compact $\mathbf{M}^{c}(X)$, qui vérifie $\mathbf{M}^{c}(X)=\mathbf{M}(X)$, si $X$ est un schéma projectif. On a, pour tout schéma $X$ lisse de dimension $n$, la relation ([V-TCM] 4.3.2.)

$$
\mathbf{M}(X)^{*}=\mathbf{M}^{c}(X)(-n)[-2 n]
$$

1.3.5. Triangles remarquables

Dans la catégorie $\mathbf{D M}_{\mathrm{gm}}(k)$ des motifs géométriques, nous utiliserons les triangles remarquables $([\mathrm{V}-\mathrm{TCM}])$ suivants :

(1.3.5.1) Gysin. Si Z est un sous-schéma fermé lisse, partout de codimension $c$, d'un schéma lisse $\mathrm{X}$,

$$
\mathbf{M}(X-Z) \rightarrow \mathbf{M}(X) \rightarrow \mathbf{M}(Z)(c)[2 c] \rightarrow \mathbf{M}(X-Z)[1] .
$$

(1.3.5.2) Localisation à support compact. Si $Z$ est un sous-schéma fermé de $X$,

$$
\mathbf{M}^{c}(Z) \rightarrow \mathbf{M}^{c}(X) \rightarrow \mathbf{M}^{c}(X-Z) \rightarrow \mathbf{M}^{c}(Z)[1]
$$


(1.3.5.3) Gysin généralisé. Si $X$ est un schéma lisse équidimensionnel de dimension $n$ et $Z$ est un sous-schéma fermé de $X$,

$$
\mathbf{M}(X-Z) \rightarrow \mathbf{M}(X) \rightarrow \mathbf{M}^{c}(Z)^{*}(n)[2 n] \rightarrow \mathbf{M}(X-Z)[1] .
$$

(1.3.5.4) Eclatement. Si $Z$ est un sous-schéma fermé d'un schéma $X$ et $p_{Z}: X_{Z} \rightarrow X$ est éclatement de $Z$ dans $X$,

$$
\mathbf{M}\left(p_{Z}^{-1}(Z)\right) \rightarrow \mathbf{M}\left(X_{Z}\right) \oplus \mathbf{M}(Z) \rightarrow \mathbf{M}(X) \rightarrow \mathbf{M}\left(p_{Z}^{-1}(Z)\right)[1] .
$$

On remarquera que le triangle de Gysin généralisé est obtenu par dualité à partir du triangle de localisation à support compact.

\subsection{Changements de base}

Proposition 1.4.1. Toute extension séparable de corps $\sigma: k \hookrightarrow K$ induit un foncteur de changement de base

$$
\begin{array}{ccc}
\sigma^{s}: \operatorname{Shv}_{N i s}(\operatorname{Smcor}(k)) & \rightarrow & \operatorname{Shv}_{N i s}(\operatorname{Smcor}(K)) \\
F & \mapsto & F_{K}
\end{array}
$$

envoyant, pour tout schéma lisse $X$, le faisceau $\mathbf{Z}_{t r}(X)$ sur le faisceau $\mathbf{Z}_{t r}\left(X_{K}\right)$ avec $X_{K}=$ $X \times \times_{\operatorname{Spec}(k)} \operatorname{Spec}(K)$.

Le foncteur $\sigma^{s}$ est exact.

Demonstration : le foncteur extension des scalaires $\alpha_{K}: \operatorname{Sm}(k) \rightarrow \operatorname{Sm}(K)$, défini par $X \mapsto$ $X \times_{\operatorname{Spec}(k)} \operatorname{Spec}(K)$ respecte les correspondances finies ([MVW] 1.12), est continu et permet de construire un foncteur changement de base. Par construction, on a pour tout faisceau avec transferts $F$ sur $\operatorname{Sm}(k)$ et tout schéma $Y$ lisse sur $K$

$$
F_{K}(Y)=\underset{(X, f)}{\lim _{(X)}} F(X)
$$

où la limite est prise sur la catégorie des couples $(X, f)$, où $X$ est un schéma lisse sur $k$ et $f$ une correspondance de $Y$ vers $X_{K}$. Comme l'extension est séparable, un tel $Y$ est limite projective filtrante de variétés lisses de type fini sur $k$, reliées par des morphismes affines : le foncteur $F \mapsto F_{K}$ est exact.

Puisque le foncteur $\sigma^{s}$ préserve l'invariance d'homotopie et le motif de Tate, qu'il est compatible au produit, il induit un foncteur de changement de base

$$
\begin{aligned}
& \sigma_{K}: \mathbf{D M}^{-}(k) \rightarrow \mathbf{D M}^{-}(K) \\
& \mathbf{M} \mapsto \mathbf{M}_{K}
\end{aligned}
$$

envoyant pour tout schéma $X$ le motif $\mathbf{M}(X)$ sur le motif $\mathbf{M}\left(X_{K}\right)$.

Remarque 1.4.2. Si l'extension $\sigma: k \hookrightarrow K$ est finie, alors $\operatorname{Spec} K$ définit un objet de $\mathbf{D M}^{-}(k)$ et le foncteur de changement de base admet un adjoint à gauche $\sigma_{K}^{*}$ induit par le foncteur de $\operatorname{Sm}(K)$ dans $\operatorname{Sm}(k)$, qui à $Y$ associe $Y$; plus précisément, si $\mathbf{M}$ est un objet de $\mathbf{D} \mathbf{M}^{-}(k)$, alors $\sigma_{K}^{*} \circ \sigma_{K}(\mathbf{M}) \simeq \mathbf{M} \otimes \mathbf{M}(\operatorname{Spec} K)$, que l'on note plus simplement $\mathbf{M} \otimes K$.

En [LW09] nous avons défini pour tout corps de caractéristique 0 un ind-motif de De Rham, limite inductive de complexes motiviques. Nous avons besoin du résultat suivant

Lemme 1.4.3. Si $k \hookrightarrow K$ est une extension de corps de caractéristique 0, le foncteur changement de base respecte les motifs de De Rham.

Demonstration : le foncteur de changement de base respectant les faisceaux avec transferts, il suffit de vérifier que le foncteur de changement de base $\widetilde{\operatorname{Sm} k}_{A b} \rightarrow \widetilde{\operatorname{Sm} K}_{A b}$ envoie le faisceau des $k$ différentielles de Kähler sur le faisceau des $K$-différentielles de Kähler. Localement, c'est la formule de changement de base des différentielles ([EGA IV] 16.6.4). 


\subsection{La filtration par les poids de Bondarko}

Utilisant la construction de la catégorie des motifs géométriques à partir de la catégorie des complexes bornés de schémas projectifs de $\operatorname{Smcor}(k)$, Bondarko munit la catégorie $\mathbf{D M}_{\mathrm{gm}}(k)$ d'une structure différentielle graduée [Bo09], la graduation sur les morphismes étant induite par celle du complexe de Suslin, plus précisément par le complexe cubique de Suslin ([Bo09] Ch.1). Cela lui permet de munir $\mathbf{D M}_{\mathrm{gm}}(k)$ d'une structure à poids ou pondérale ("weight structure"), à savoir

Theoreme 1.5.1. ([Bo10] 1.1.1. et 6.5.3.)

Il existe deux sous-catégories $\mathbf{D}^{w \geq 0}$ et $\mathbf{D}^{w \leq 0}$ de $\mathbf{D M}_{\mathrm{gm}}(k)$ telles que

(i) $\mathbf{D}^{w \geq 0}$ et $\mathbf{D}^{w \leq 0}$ sont additives et Karoubiennes;

(ii) semi-invariance par translation : $\quad \mathbf{D}^{w \geq 0} \subset \mathbf{D}^{w \geq 0}[1]$ et $\mathbf{D}^{w \leq 0}[1] \subset \mathbf{D}^{w \leq 0}$;

(iii) orthogonalité : pour tout objet $\mathbf{M}$ de $\mathbf{D}^{w \geq 0}$ et tout objet $\mathbf{L}$ de $\mathbf{D}^{w \leq 0}[1]$, on a

$$
\operatorname{Hom}_{\mathbf{D M}_{\mathrm{gm}}(k)}(\mathbf{M}, \mathbf{L})=\{0\} ;
$$

(iv) décomposition en poids : pour tout motif géométrique $\mathbf{M}$, il existe un triangle distingué

$$
\mathbf{M} \rightarrow \mathbf{A} \rightarrow \mathbf{B} \rightarrow \mathbf{M}[1]
$$

avec $\mathbf{A}$ objet de $\mathbf{D}^{w \leq 0}$ et $\mathbf{B}$ objet de $\mathbf{D}^{w \geq 0}$.

REMARQUeS :

1.5.2. Les catégories $\mathbf{D}^{w \geq 0}$ et $\mathbf{D}^{w \leq 0}$ sont construites respectivement à partir des classes de complexes bornés de schémas projectifs lisses de $\operatorname{Smcor}(k)$ qui sont concentrés en degrés positifs ou négatifs. Plus précisément, si l'on note $\mathbf{D}^{w=0}=\mathbf{D}^{w \leq 0} \cap \mathbf{D}^{w \leq 0}$, la catégorie des motifs de poids 0 , Bondarko l'identifie à la catégorie de Grothendieck des motifs de Chow ([Bo10] 6.2) en utilisant les résultats de Friedlander et Voevodsky [FV]. Ainsi les sommes finies de motifs de schémas projectifs lisses et leurs facteurs directs, comme les $\mathbf{Z}(q)[2 q]$, sont de poids 0 . Sur un corps qui vérifie la résolution des singularités, on montre en utilisant les triangles de Gysin que les schémas lisses sont de poids positifs ([Bo09] 6.2.1).

1.5.3. La décomposition en poids (iv) n'est pas unique. Néanmoins Bondarko montre que l'on peut fixer a priori pour chaque motif géométrique $\mathbf{M}$ des décompositions

$$
\mathbf{M}[i] \rightarrow \mathbf{M}^{w \leq i} \rightarrow \mathbf{M}^{w \geq i+1} \rightarrow \mathbf{M}[i+1]
$$

et construire un complexe ([Bo10] 2.2) dit complexe des poids

$$
\cdots \mathbf{M}^{(i-1)} \stackrel{p_{i-1}}{\longrightarrow} \mathbf{M}^{(i)} \stackrel{p_{i}}{\longrightarrow} \mathbf{M}^{(i+1)} \rightarrow \cdots
$$

avec $([$ Bo10] 1.5.6)

$$
\begin{aligned}
& \mathbf{M}^{(i)} \simeq \text { cône }\left(\begin{array}{c}
\mathbf{M}^{w \leq i}[-1] \quad \rightarrow \quad \mathbf{M}^{w \leq i-1}
\end{array}\right) \\
& \simeq \text { cône }\left(\mathbf{M}^{w \geq i+1}[-1] \rightarrow \mathbf{M}^{w \geq i} \quad\right)
\end{aligned}
$$

et $\mathbf{M}^{(i)}$ est un motif de poids 0 . Un tel complexe n'est pas unique et sa construction n'est donc pas fonctorielle, mais, lorsqu'on lui applique un foncteur cohomologique, il fournit une suite spectrale qui est fonctorielle à partir de $E_{2}$ (cf ci-dessous 1.5.9.).

Il est possible de donner une description explicite d'un complexe des poids du motif $\mathbf{M}(X)$, lorsque $X$ est un schéma lisse et quasi-projectif sur $k$. Les notations sont celles de [D71], Ch.3.

Proposition 1.5.4. Soit $X$ un schéma lisse et quasi-projectif sur $k$, plongé dans un schéma projectif lisse $\bar{X}$, tel que le schéma complémentaire $Y=\bar{X}-X$ soit un diviseur à croisements normaux et à composantes irréductibles lisses $Y=\cup_{i=0}^{N-1} Y_{i}$. Désignons par $Y^{j}$ (resp. $\widetilde{Y^{j}}$ ) la réunion (resp. somme disjointe) des intersections $j$ à $j$ des $Y_{i}$. On pose $\widetilde{Y^{0}}=Y^{0}=\bar{X}$ et $\widetilde{Y}=\widetilde{Y^{1}}=\bigsqcup_{0 \leq i \leq N-1} Y_{i}$. Le complexe $\left(\mathbf{M}(X)^{(j)}\right)$ tel que, en degré $j$,

$$
\mathbf{M}(X)^{(j)}=\left\{\begin{array}{lc}
\mathbf{M}\left(\widetilde{Y^{j}}\right)(j)[2 j] & \text { si } 0 \leq j \leq N \\
0 & \text { sinon }
\end{array}\right.
$$

et dont les différentielles sont les sommes alternées des morphismes induits par les inclusions de $\widetilde{Y^{j+1}}$ dans $\widetilde{Y^{j}}$ est un complexe des poids de $\mathbf{M}(X)$. 
Demonstration : on a, pour l'inclusion $X \hookrightarrow \bar{X}$, le triangle de Gysin généralisé, où $n=\operatorname{dim}(X)$

$$
\mathbf{M}(X) \rightarrow \mathbf{M}(\bar{X}) \rightarrow \mathbf{M}(Y)^{*}(n)[2 n] \rightarrow \mathbf{M}(X)[1]
$$

Le motif $\mathbf{M}(\bar{X})$ est de poids 0 et si l'on prouve que le motif $\mathbf{M}(Y)^{*}(n)[2 n]$ est de poids positif, alors le triangle (1.5.5) est une décomposition en poids de $\mathbf{M}(X)$ et des manipulations élémentaires dans les catégories pondérales ([Bo10], lemme 1.5.4) prouvent que l'on peut choisir comme complexe des poids de $\mathbf{M}(X)$ un complexe des poids de $\mathbf{M}(Y)^{*}(n)[2 n]$ décalé d'un degré et augmenté de $\mathbf{M}(\bar{X})$ en degré 0 . Plus précisément, on obtient

$$
\begin{aligned}
& \left.\mathbf{M}(X)^{(j)}=\mathbf{M}(Y)^{*}(n)[2 n]\right)^{(j-1)} \quad \text { si } 1 \leq j \\
& \mathbf{M}(X)^{(0)}=\mathbf{M}(\bar{X}) .
\end{aligned}
$$

Ainsi il est équivalent de calculer les poids de $\mathbf{M}(X)$ ou de $\mathbf{M}(Y)^{*}(n)[2 n]$. Tout repose sur le lemme Lemme 1.5.6. Soit $Q=\cup_{i=0}^{N-1} Q_{i}$ un diviseur à croisements normaux, à $N$ composantes irréductibles lisses, d'une variété lisse $P$ de dimension $n$. Un complexe des poids de $\mathbf{M}=\mathbf{M}(Q)^{*}(n)[2 n]$ est

$$
\mathbf{M}^{(i)}=\left\{\begin{array}{lc}
\mathbf{M}\left(\widetilde{Q^{i+1}}\right)(i+1)[2 i+2] & \text { si } 0 \leq i \leq N-1 \\
0 & \text { sinon }
\end{array}\right.
$$

Demonstration : on procède par récurrence sur le nombre $N$ de composantes de $Q$. Si $N=1$, le schéma $Q$ est projectif lisse de dimension $n-1$ et le motif $\mathbf{M}(Q)^{*}(n)[2 n]=\mathbf{M}(Q)(1)[2]$ est de poids 0 .

Supposons le lemme démontré pour $N$ composantes et considérons le schéma $Q=\cup_{i=0}^{N} Q_{i} \hookrightarrow P$. Soit $Z=\cup_{i=0}^{N-1} Q_{i}$ le diviseur à croisements normaux à $N$ composantes. Le triangle de localisation à support compact (1.3.5.2) pour l'inclusion $Z \hookrightarrow Q$ est

$$
\mathbf{M}(Z) \rightarrow \mathbf{M}(Q) \rightarrow \mathbf{M}^{c}(Q-Z) \rightarrow \mathbf{M}(Z)[1]
$$

Le schéma $Q-Z=Q_{N}-\left(Q_{N 0} \cup \ldots \cup Q_{N N-1}\right)$, avec $Q_{N i}=Q_{N} \cap Q_{i}$, est lisse et son complexe des poids est connu par récurrence. Par dualité, on a

$$
\mathbf{M}^{c}(Q-Z)^{*}=\mathbf{M}(Q-Z)(-n+1)[-2 n+2] .
$$

Le dual du triangle (1.5.7) devient après torsion par $\mathbf{Z}(n)[2 n]$

$$
\mathbf{M}(Q-Z)(1)[2] \rightarrow \mathbf{M} \rightarrow \mathbf{M}(Z)^{*}(n)[2 n] \rightarrow \mathbf{M}(Q-Z)(1)[3] .
$$

En appliquant à nouveau le lemme 1.5.4 de [Bo10], le triangle (1.5.8.) fournit en chaque poids un triangle distingué qui est scindé, car chacune des extrémités est somme de motifs purs de poids 0 ([Bo10] Prop. 1.3.1.(7)). Par conséquent le complexe des poids de $\mathbf{M}$ est la somme des deux complexes des poids de $\mathbf{M}_{1}=\mathbf{M}(Q-Z)(1)[2]$ et de $\mathbf{M}_{2}=\mathbf{M}(Z)^{*}(n)[2 n]$. On a en degré $i$ les intersections $(i+1)$ à $(i+1)$ des $Q_{i}$, tordues par $\mathbf{Z}(i+1)[2 i+2]$; dans $\mathbf{M}_{1}$ viennent celles où apparaît $Q_{N}$, dans $M_{2}$ celles où la composante $Q_{N}$ n'apparaît pas.

1.5.9. Pour tout foncteur cohomologique $H$ de $\mathbf{D M}_{\mathrm{gm}}(k)$ vers une catégorie abélienne $A$ et tout entier $i$, on pose

$$
\left(W_{i} H\right)(\mathbf{M})=\operatorname{Im}\left(H\left(w_{\leq i} \mathbf{M}\right) \rightarrow H(\mathbf{M})\right)
$$

où l'on a noté $w_{\leq i} \mathbf{M}=\mathbf{M}^{w \leq i}[-i]$. On obtient ainsi une filtration croissante sur $H(\mathbf{M})$, qui ne dépend pas du choix de $\mathbf{M}^{w \leq i}$, appelée filtration par le poids. D'autre part, on dispose de la suite spectrale associée au complexe des poids de $\mathbf{M}$

$$
E_{1}^{p q}(\mathbf{M})=H^{q}\left(\mathbf{M}^{(-p)}\right) \Rightarrow H^{p+q}(\mathbf{M}) .
$$

Le terme $E_{1}$ dépend du choix du complexe des poids, mais pas le terme $E_{2}$ et la suite est fonctorielle en $\mathbf{M}$ à partir de $E_{2}$ (cf [Bo10], Th 2.4.1).

Pour un motif $\mathbf{M}$, notons $\operatorname{Gr}_{i}^{W} H(\mathbf{M})=\left(W_{i} H\right)(\mathbf{M}) /\left(W_{i-1} H\right)(\mathbf{M})$; on constate que

$$
\begin{aligned}
\left(W_{i} H\right)(\mathbf{M}[-p]) & =\operatorname{Im}\left(H\left(w_{\leq i}(\mathbf{M}[-p])\right) \rightarrow H(\mathbf{M}[-p])\right) \\
& =\operatorname{Im}\left(H\left(\left(w_{\leq i-p} \mathbf{M}\right)[-p]\right) \rightarrow H(\mathbf{M}[-p])\right) \\
& =\operatorname{Im}\left(H^{p}\left(w_{\leq i-p} \mathbf{M}\right) \rightarrow H^{p}(\mathbf{M})\right) \\
& =\left(W_{i-p} H^{p}\right)(\mathbf{M})
\end{aligned}
$$

d'où le décalage habituel $\mathrm{Gr}_{i}^{W} H(\mathbf{M}[-p])=\mathrm{Gr}_{i-p}^{W} H^{p}(\mathbf{M})(\operatorname{cf}[\mathrm{D} 71])$.

Une transformation naturelle entre deux foncteurs cohomologiques préserve les filtrations par le poids. 


\section{Réalisation de De Rham}

\subsection{Construction}

En [LW09], nous avons muni les faisceaux $X \mapsto \Omega_{X / k}^{n}(X)$ de transferts et défini un ind-complexe motivique $\Omega$ qui représente la cohomologie de De Rham, dans le sens que pour tout schéma $X$ lisse sur $k$, on a

$$
\mathbb{H}_{Z a r}^{p}\left(X, \Omega_{X / k}\right)=\operatorname{Hom}_{\mathbf{D M}(k)}(\mathbf{M}(X), \boldsymbol{\Omega}[p])
$$

où $\Omega_{X / k}$ est le complexe de De Rham de $X$ et l'on a plongé la catégorie $\mathbf{D M}^{-}(k)$ dans la catégorie des complexes motiviques non bornés $\mathbf{D M}(k)$ de Cisinski et Déglise [CD09]. On généralise la cohomologie de de Rham à tous les motifs en posant

Definition 2.1.1. La réalisation de De Rham de tout complexe motivique $\mathbf{M}$ est le $k$-espace vectoriel gradué

$$
\mathbf{H}_{D R}^{\cdot}(\mathbf{M})=\oplus_{p \geq 0} \mathbf{H}_{D R}^{p}(\mathbf{M})=\oplus_{p \geq 0} \mathbf{H}_{D R}(\mathbf{M}[-p])
$$

associé au foncteur cohomologique $\mathbf{H}_{D R}$ de $\mathbf{D} \mathbf{M}^{-}(k)$ dans la catégorie des $k$-espaces vectoriels

$$
\mathbf{H}_{D R}(\mathbf{M})=\operatorname{Hom}_{\mathbf{D M}(k)}(\mathbf{M}, \boldsymbol{\Omega}) .
$$

Cette définition coïncide avec la définition de [LW09] où nous tronquions le complexe de De Rham en vertu du lemme suivant.

Lemme 2.1.2. Pour tout complexe motivique $\mathbf{M}$ de $\mathbf{D M}^{-}(k)$, on a pour tout entier $i$, les isomorphismes

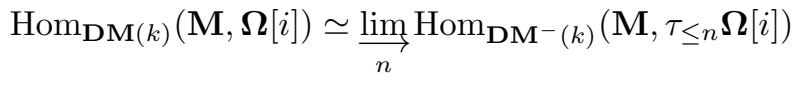

où $\tau_{\leq n}$ est le foncteur de filtration canonique à droite.

Demonstration : la propriété ci-dessus étant stable par quasi-isomorphisme, décalage et cône, il suffit par le lemme 9.3 de [MVW] de vérifier que pour toute famille de schémas lisses $\left(X_{\alpha}\right)_{\alpha \in A}$ on a

$$
\prod_{\alpha \in A} \underset{n}{\lim _{\longrightarrow}} \operatorname{Hom}_{D^{-}}\left(\mathbf{Z}_{t r}\left(X_{\alpha}\right), \tau_{\leq n} \boldsymbol{\Omega}[i]\right)=\underset{n}{\lim } \prod_{\alpha \in A} \operatorname{Hom}_{D^{-}}\left(\mathbf{Z}_{t r}\left(X_{\alpha}\right), \tau_{\leq n} \boldsymbol{\Omega}[i]\right)
$$

ce qui provient du fait que les complexes $\mathbf{Z}_{t r}\left(X_{\alpha}\right)$ sont concentrés en degré zéro, les complexes $\tau_{\leq n} \boldsymbol{\Omega}[i]$ sont bornés et la suite de droite est stationnaire pour $n>i$.

Comme la cohomologie de de Rham des schémas projectifs lisses est de dimension finie, le principe (0.2) implique que, restreinte aux motifs géométriques, la réalisation de De Rham est un $k$-espace vectoriel de dimension finie.

Lemme 2.1.3. Le morphisme de faisceaux de Nisnevich dlog: $\mathcal{O}^{*} \rightarrow \Omega^{1}$ commute aux transferts.

Demonstration : en [LW09], nous avons défini le transfert sur le faisceau des différentielles $\Omega^{1}$ en passant aux différentielles de Zariski $\Omega^{Z a r}$, au sens de [K73]. Soit $Z$ une correspondance d'un schéma lisse irréductible $X$ vers un schéma lisse $Y$. Comme chez Suslin et Voedvodsky [SV96] on se ramène au cas où $Z$ est la normalisée de $X$ dans une extension galoisienne finie du corps des fonctions $K(X)$ de $X$, de groupe de Galois $G=\operatorname{Gal}(K(Z) / K(X))$. On doit démontrer la commutativité du diagramme suivant :

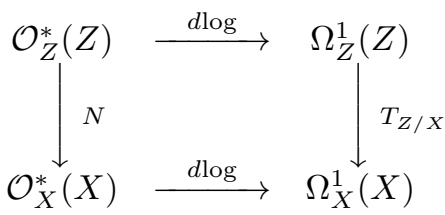

où $N$ est la norme $N(f)=\prod_{\sigma \in G} \sigma(f)$ et $T_{Z / X}$ le transfert qui a été défini en (loc. cit) comme la composée

$$
\Omega_{Z}^{1}(Z) \stackrel{\alpha_{Z}}{\longrightarrow} \Omega_{Z}^{Z a r}(Z) \stackrel{\sum_{\sigma \in G} \sigma^{*}}{\longrightarrow} \Omega_{X}^{Z a r}(X) \stackrel{\alpha_{X}^{-1}}{\simeq} \Omega_{X}^{1}(X) .
$$


Ici, pour tout schéma $U$, on désigne par $\Omega_{U}^{Z a r}$ le faisceau bidual (au sens de $\mathcal{O}_{U}$-module dans la topologie de Zariski sur $U$ ) de $\Omega_{U}^{1}$ et $\alpha_{U}: \Omega_{U}^{1} \rightarrow \Omega_{U}^{Z a r}$ l'application canonique qui consiste à quotienter par la torsion. Le morphisme $\alpha_{U}$ est un isomorphisme quand le schéma $U$ est lisse.

Si le schéma $Z$ est lisse, le transfert coïncide avec la trace $\operatorname{Tr}=\sum_{\sigma \in G} \sigma^{*}$ et le lemme est la traduction de la propriété $d \log \circ N=T r \circ d \log$.

Si le schéma $Z$ n'est que normal, il suffit de vérifier que cette propriété n'est pas altérée par le passage au bidual. En remarquant que pour toute fonction inversible $f$ de $\mathcal{O}_{Z}^{*}(Z)$, la forme $\omega=d \log f$ vérifie la propriété caractéristique des différentielles de Zariski sur un schéma normal, à savoir

$$
P(Z): \forall x \in Z, \forall D \in \operatorname{Der}_{k}\left(\mathcal{O}_{x}, \mathcal{O}_{x}\right), \tilde{D}(\omega) \in \mathcal{O}_{x}
$$

où $\operatorname{Der}_{k}\left(\mathcal{O}_{x}, \mathcal{O}_{x}\right)$ est l'espace des $k$-dérivations de l'anneau local $\mathcal{O}_{x}$ dans lui-même et $\tilde{D}: \Omega_{\mathcal{O}_{x} / k}^{1} \rightarrow$ $\mathcal{O}_{x}$ est le morphisme $\mathcal{O}_{x^{-}}$linéaire canoniquement associé à la différentielle $D$.

Or on a, pour toute fonction $f \in \mathcal{O}_{Z}^{*}(Z)$ et tout point $x$ de $Z$,

$$
\tilde{D}(d \log f)=\tilde{D}\left(\frac{d f_{x}}{f_{x}}\right)=\frac{D f_{x}}{f_{x}} \in \mathcal{O}_{x}
$$

Ce morphisme $d$ log induit le morphisme de $\mathbf{D M}^{-}(k)$

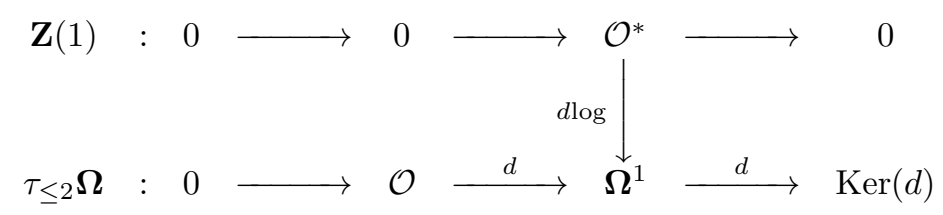

qui produit un générateur de $\mathbf{H}_{D R}^{0}(\mathbf{Z}(1))$. On vérifie que l'image de $d$ log se trouve dans $\mathrm{Ker} d$, d'où un morphisme $\mathbf{Z}(1) \rightarrow \tau_{\leq 1} \Omega$.

Un générateur de $\mathbf{H}_{D R}^{0}(\mathbf{Z}(q))$, lorsque $q$ est un entier positif, est induit par le morphisme issu du produit sur $\Omega$ [LW09]

$$
\mathbf{Z}(q)=\mathbf{Z}(1)^{\otimes q} \stackrel{d \log ^{\otimes q}}{\longrightarrow}(\boldsymbol{\Omega})^{\otimes q} \rightarrow \boldsymbol{\Omega} .
$$

Ces morphismes permettent de définir des classes de Chern

$$
c_{D R}^{p, q}: H^{p, q}(\mathbf{M})=\operatorname{Hom}_{\mathbf{D M}^{-}(k)}(\mathbf{M}, \mathbf{Z}(q)[p]) \rightarrow \mathbf{H}_{D R}^{p}(\mathbf{M})
$$

pour tout complexe motivique $\mathbf{M}$ de $\mathbf{D} \mathbf{M}^{-}$.

Le produit de [LW09] fournit aussi pour toute paire de complexes motiviques $\mathbf{M}_{1}$ et $\mathbf{M}_{2}$ de $\mathbf{D} \mathbf{M}^{-}(k)$ un accouplement

$$
\mathbf{H}_{D R}^{p_{1}}\left(\mathbf{M}_{1}\right) \otimes \mathbf{H}_{D R}^{p_{2}}\left(\mathbf{M}_{2}\right) \rightarrow \mathbf{H}_{D R}^{p_{1}+p_{2}}\left(\mathbf{M}_{1} \otimes \mathbf{M}_{2}\right)
$$

\subsection{Filtration par les poids}

La filtration par les poids est induite sur le foncteur cohomologique par la structure de poids définie par Bondarko [Bo10]

$$
W_{i} \mathbf{H}_{D R}(\mathbf{M})=\operatorname{Im}\left(\mathbf{H}_{D R}\left(w_{\leq i} \mathbf{M}\right) \rightarrow \mathbf{H}_{D R}(\mathbf{M})\right)
$$

qui induit la filtration sur $\mathbf{H}_{D R}^{p}(\mathbf{M})$

$$
W_{i} \mathbf{H}_{D R}^{p}(\mathbf{M})=W_{i+p} \mathbf{H}_{D R}(\mathbf{M}[-p])
$$

Proposition 2.2.2. Si $X$ est un schéma lisse quasi-projectif sur $k$, la filtration $W$ sur $\mathbf{H}_{D R}(\mathbf{M}(X))$ coïncide avec la filtration par le poids classique de la cohomologie de $X$. 
Demonstration : nous choisissons comme référence les travaux de Deligne en théorie de Hodge, plus précisément ([D71] Ch.3), repris sur un corps $k$ par Jannsen ([J90]). Les calculs, à base de pôles logarithmiques, y sont faits en cohomologie analytique mais s'adaptent en cohomologie de De Rham.

Reprenons les notations de la proposition 1.5.4 : le schéma $X$ étant lisse et quasi-projectif sur $k$ comme dans (loc. cit)(3.2.1), on le plonge dans un schéma projectif lisse $\bar{X}$, tel que le schéma complémentaire $Y=\bar{X}-X$ soit un diviseur à croisements normaux et à composantes irréductibles lisses $Y=\cup_{i=0}^{N-1} Y_{i}$. Comme en (loc. cit.), on désigne par $Y^{j}$ (resp. $\widetilde{Y^{j}}$ ) la réunion (resp. somme disjointe) des intersections $j$ à $j$ des $Y_{i}$. On pose $\widetilde{Y^{0}}=Y^{0}=\bar{X}$ et $\widetilde{Y}=\widetilde{Y^{1}}=\coprod_{0 \leq i \leq N-1} Y_{i}$. On a vu que $\mathbf{M}(X)^{(0)}=\mathbf{M}(\bar{X})$ et $\mathbf{M}(X)^{(k)}=\mathbf{M}\left(\widetilde{Y^{k}}\right)(k)[2 k]$ pour $1 \leq k \leq N-1$.

La suite spectrale de Bondarko ([Bo10] 2.4.1.)

$$
E_{1}^{p q}(\mathbf{M}(X))=\mathbf{H}_{D R}^{q}\left(\mathbf{M}(X)^{(-p)}\right) \Rightarrow \mathbf{H}_{D R}^{p+q}(\mathbf{M}(X))
$$

coïncide alors avec la suite spectrale de Jannsen ([J90], I3.) ou de Deligne ([D71] 3.2.7) lorsque $k=\mathbf{C}$

$$
{ }_{W} E_{1}^{p, q}(X)=\mathbb{H}^{q+2 p}\left(\widetilde{Y^{-p}}, \Omega_{\widetilde{Y^{-p}}}\right) \otimes_{k} \mathbf{H}_{D R}^{0}(\mathbf{Z}(-p)) .
$$

On en déduit qu'elle dégénère en $E_{2}$ (cf. corollaire 3.2.13, [D71], lorsque $k=\mathbf{C}$ ) ainsi que l'isomorphisme

$$
E_{2}^{p, q}(\mathbf{M}(X))=\operatorname{Gr}_{q}^{W} \mathbf{H}_{D R}^{p+q}(\mathbf{M}(X))
$$

qui tient compte du décalage.

\subsection{Filtration de Hodge}

Dans la catégorie dérivée $D\left(\operatorname{Shv}_{N i s}(\operatorname{Smcor}(k))\right)$ des complexes de faisceaux avec transferts, la filtration bête ou troncature à gauche

$$
\left(\sigma_{\geq p} \boldsymbol{\Omega}\right)^{q}(X)=\left\{\begin{array}{lcc}
\Omega_{X / k}^{q}(X) & \text { si } & p \leq q \\
0 & \text { sinon } &
\end{array}\right.
$$

fournit pour tout complexe borné $K$ de $D_{N i s}^{-}(\operatorname{Smcor}(k))$ une suite spectrale

$$
E_{1}^{p, q}(K)=\operatorname{Hom}_{D\left(\operatorname{Shv}_{N i s}(\operatorname{Smcor}(k))\right)}\left(K, \boldsymbol{\Omega}^{p}[q]\right) \Rightarrow \operatorname{Hom}_{D\left(\operatorname{Shv}_{N i s}(\operatorname{Smcor}(k))\right)}(K, \boldsymbol{\Omega}[p+q]) .
$$

Cette suite spectrale est non seulement fonctorielle en $K$ mais, de plus, comme pour la suite spectrale des poids de Bondarko, elle est fonctorielle sur $\mathbf{D M}^{-}(k)$ à partir du terme $E_{2}$ :

Proposition 2.3.2. Si $f: K \rightarrow K^{\prime}$ est une $\mathbf{A}^{1}$-équivalence de complexes de faisceaux avec transferts dans $D_{N i s}^{-}(\operatorname{Smcor}(k))$, alors on a, pour tout $r \geq 2$ et tout couple d'entiers $(p, q)$, des isomorphismes

$$
E_{r}^{p, q}\left(K^{\prime}\right) \simeq E_{r}^{p, q}(K)
$$

Demonstration : il suffit de montrer que, pour tout couple d'entiers $(p, q)$, on a un isomorphisme

$$
E_{2}^{p, q}\left(K^{\prime}\right) \simeq E_{2}^{p, q}(K)
$$

compatible aux différentielles. En adaptant la démonstration du lemme d'homotopie aux bicomplexes, on se ramène à prouver que pour tout schéma $X$ lisse sur $k$, la projection $\pi_{X}: X \times_{k} \mathbf{A}^{1} \rightarrow X$ induit pour tout couple d'entiers $(p, q)$ un isomorphisme

$$
E_{2}^{p, q}\left(\mathbf{Z}_{t r}(X)\right) \simeq E_{2}^{p, q}\left(\mathbf{Z}_{t r}\left(X \times_{k} \mathbf{A}^{1}\right)\right)
$$

Sur le site $X_{N i s}$, considérons les complexes de faisceaux $\Omega_{X}$ et $\pi_{X *} \Omega_{X \times{ }_{k} \mathbf{A}^{1}}$. Les membres de l'isomorphisme (2.3.3) sont les termes $E_{2}$ des suites spectrales associées à la filtration bête des complexes respectifs. D'après [Ver96](4.4.3), ces suites coïncident à partir de $E_{2}$ lorsque les deux 
complexes sont homotopes. Or la décomposition des formes différentielles et la formule de projection impliquent l'isomorphisme

$$
\pi_{X *} \Omega_{X \times \mathbf{A}^{1}} \simeq \Omega_{X} \otimes \mathcal{O}_{X} \pi_{X *} \pi_{\mathbf{A}^{1}}^{-1} \Omega_{\mathbf{A}^{1}}
$$

qui permet de remonter l'homotopie induite par l'intégration

$$
\begin{aligned}
s: \quad \Omega_{\mathbf{A}^{1}}^{1} & \rightarrow \mathcal{O}_{\mathbf{A}^{1}} \\
f(t) d t & \mapsto \int_{0}^{t} f(u) d u
\end{aligned}
$$

en une homotopie entre l'application composée $\Omega_{X \times{ }_{k} \mathbf{A}^{1}} \rightarrow \Omega_{X} \rightarrow \Omega_{X \times{ }_{k}} \mathbf{A}^{1}$ et l'identité.

On a ainsi une suite spectrale $E_{r}^{p, q}(\mathbf{M}), r \geq 2$ définie pour tout complexe motivique $\mathbf{M}$. Si de plus le motif $\mathbf{M}$ est géométrique, le principe (0.2) implique que les espaces vectoriels sont de dimension finie sur $k$ et que la suite spectrale (2.3.1) d'aboutissement $\mathbf{H}_{D R}(\mathbf{M})$ dégénère et définit sur $\mathbf{H}_{D R}(\mathbf{M})$ la filtration régulière

$$
F_{\text {naive }}^{q} \mathbf{H}_{D R}^{p}(\mathbf{M})=\operatorname{Im}\left(\operatorname{Hom}_{D\left(\operatorname{Shv}_{N i s}(\operatorname{Smcor}(k))\right)}\left(\mathbf{M}, \sigma_{\geq q} \boldsymbol{\Omega}[p]\right) \rightarrow \operatorname{Hom}_{\mathbf{D M}}(\mathbf{M}, \boldsymbol{\Omega}[p])\right) .
$$

On a par construction

Proposition 2.3.5. Si M est le motif associé à un schéma $X$ lisse et projectif, la filtration $F_{\text {naive }}$ sur $\mathbf{H}_{D R}(\mathbf{M})$ est la filtration de Hodge de la cohomologie du schéma $X$.

Si le schéma lisse $X$ n'est pas projectif, c'est traditionnellement en remplaçant le complexe de de Rham par un complexe de de Rham à pôles logarithmiques, qui permet aussi de retrouver les poids, que l'on décrit la filtration de Hodge. De façon similaire, nous résolvons par les poids en remplaçant tout motif géométrique par un complexe des poids pour obtenir la filtration de Hodge.

Proposition 2.3.6. Soient $\mathbf{M}$ un motif géométrique effectif, $\mathbf{M}^{(\cdot)}$ un complexe des poids associé à M. La cohomologie du complexe total $\mathbf{K}_{D R}$ du bicomplexe $\mathrm{R} \cdot \operatorname{Hom}\left(\mathbf{M}^{(\cdot)}, \boldsymbol{\Omega}\right)$ calcule la réalisation de De Rham de $\mathbf{M}$.

Demonstration

Pour un motif géométrique effectif, on peut construire un complexe des poids borné et quitte à translater, on se ramène au cas où $\mathbf{M}$ est de poids positif ou nul. Tout complexe des poids associé à $\mathbf{M}$ s'écrit $\mathbf{M}^{(0)} \rightarrow \cdots \rightarrow \mathbf{M}^{(M)} \rightarrow 0$ avec $\mathbf{M}^{(0)} \neq 0$.

La flèche $\mathbf{M} \rightarrow \mathbf{M}^{w \leq 0}$ et l'égalité $\mathbf{M}^{(0)}=\mathbf{M}^{w \leq 0}$ induisent une flèche entre les complexes

$$
\mathbf{K}_{D R} \rightarrow \mathrm{R} \operatorname{Hom}(\mathbf{M}, \mathbf{\Omega}) .
$$

Le complexe R $\operatorname{Hom}(\mathbf{M}, \boldsymbol{\Omega})$ est muni de la filtration par les poids à la Bondarko

$$
W_{k} \mathrm{R}^{\cdot} \operatorname{Hom}(\mathbf{M}, \boldsymbol{\Omega})=\operatorname{Im}\left(\mathrm{R}^{\cdot} \operatorname{Hom}\left(w_{\leq k} \mathbf{M}, \boldsymbol{\Omega}\right) \rightarrow \mathrm{R} \cdot \operatorname{Hom}(\mathbf{M}, \boldsymbol{\Omega})\right)
$$

qui fournit une suite spectrale ${ }^{W} E_{1}^{p, q}(\mathrm{R} \operatorname{Hom}(\mathbf{M}, \boldsymbol{\Omega}))=\mathbf{H}_{D R}^{q}\left(\mathbf{M}^{(-p)}\right)$ convergeant fortement vers $\mathbf{H}_{D R}(\mathbf{M})$. D'autre part, la première filtration du bicomplexe $K_{D R}^{p, q}=\mathrm{R}^{q} \operatorname{Hom}\left(\mathbf{M}^{(-p)}, \boldsymbol{\Omega}\right)$ fournit la suite spectrale

$$
{ }^{W} E_{1}^{p, q}\left(\mathbf{K}_{D R}\right)=\mathbf{H}_{D R}^{q}\left(M^{(-p)}\right) .
$$

Puisque l'on peut choisir le complexe $\mathbf{M}^{(0)} \rightarrow \cdots \rightarrow \mathbf{M}^{(k)} \rightarrow 0$ comme complexe des poids de $w_{\leq k} \mathbf{M}$, la flèche (2.3.7) est compatible aux filtrations et induit un isomorphisme entre les suites spectrales associées.

Le théorème 3.2 , chapitre XV de [CE], permet de conclure.

Definition 2.3.8. Soit $\mathbf{M}$ un motif géométrique effectif, $\mathbf{M}^{(\cdot)}$ un complexe des poids associé. La filtration de Hodge de $\mathbf{H}_{D R}(\mathbf{M})$ est induite par la filtration du bicomplexe

$$
F^{j}\left(\mathrm{R}^{q} \operatorname{Hom}\left(\mathbf{M}^{(-p)}, \boldsymbol{\Omega}\right)\right)=\operatorname{Im}\left(\mathrm{R}^{q} \operatorname{Hom}\left(\mathbf{M}^{(-p)}, \sigma \geq j \boldsymbol{\Omega}\right) \rightarrow \mathrm{R}^{q} \operatorname{Hom}\left(\mathbf{M}^{(-p)}, \boldsymbol{\Omega}\right)\right)
$$

et

$$
F^{j} \mathrm{R} \operatorname{Hom}(\mathbf{M}, \boldsymbol{\Omega})=\operatorname{Im}\left(F^{j} \mathrm{R} \cdot \operatorname{Hom}\left(\mathbf{M}^{(\cdot)}, \boldsymbol{\Omega}\right) \rightarrow \mathrm{R} \cdot \operatorname{Hom}(\mathbf{M}, \boldsymbol{\Omega})\right)
$$

Remarque : nous verrons en (4.2.4) que ces définitions ne dépendent pas des choix, notamment de celui du complexe des poids. 


\subsection{Motif de De Rham analytique}

En munissant la catégorie $\operatorname{Smcor}(\mathbf{C})$ des schémas algébriques complexes lisses et des correspondaces finies de la topologie analytique, on fait les mêmes constructions qu'en topologie étale pour définir une catégorie triangulée $\mathbf{D M}_{a n}^{-}$. Rappelons que le théorème d'invariance d'homotopie (1.3.3.1) n'est plus valable dans ce cadre. Le changement de topologie définit un foncteur

$$
\begin{array}{ccc}
t_{a n}: \mathbf{D M}^{-}(\mathbf{C}) & \rightarrow \mathbf{D M}_{a n}^{-} \\
\mathbf{M} & \mapsto & \mathbf{M}_{a n}
\end{array}
$$

D'après [GAGA], ce foncteur envoie le faisceau structural $X \mapsto \Gamma\left(X, \mathcal{O}_{X}\right)$ sur le faisceau structural des fonctions holomorphes et les différentielles de Kähler sur les différentielles holomorphes, le ind-motif $\boldsymbol{\Omega}$ vers le ind-motif noté $\boldsymbol{\Omega}_{a n}$ dont le $n^{\text {ième }}$-terme est le faisceau $X \mapsto \Omega_{X}^{n, h}(X)$ des $n$-différentielles holomorphes.

Proposition 2.4.1 Le foncteur cohomologique $\mathbf{M} \mapsto \operatorname{Hom}_{\mathbf{D M}_{a n}}\left(\mathbf{M}_{a n}, \boldsymbol{\Omega}_{a n}\right)$ de la catégorie des motifs géométriques vers la catégorie des $\mathbf{C}$-espaces vectoriels coïncide avec la réalisation de De Rham.

Demonstration : Le foncteur $t_{a n}$ induit pour tout motif $\mathbf{M}$ géométrique de $\mathbf{D M}_{\mathrm{gm}}(\mathbf{C})$ un morphisme

$$
\operatorname{Hom}_{\mathbf{D M}(\mathbf{C})}(\mathbf{M}, \boldsymbol{\Omega}) \rightarrow \operatorname{Hom}_{\mathbf{D M}}\left(\mathbf{M}_{a n}, \boldsymbol{\Omega}_{a n}\right) .
$$

Si $\mathbf{M}=\mathbf{M}(X)$ est le motif d'un schéma lisse projectif, ce morphisme est un isomorphisme d'après [GAGA]. On conclut en rappelant que de tels $\mathbf{M}(X)$ engendrent $\mathbf{D M}_{\mathrm{gm}}(\mathbf{C})$.

\section{Réalisation de Betti}

Cette partie est largement inspirée des travaux de Suslin et Voevodsky [SV96] et a été résumée en [L08].

\subsection{Réalisation topologique des motifs sur C.}

Nous travaillons sur le site $\operatorname{Sm}(\mathbf{C})$ des schémas lisses sur le corps des nombres complexes, muni de la topologie de Nisnevich, et sur le site $C W$ des espaces topologiques réels admettant une triangulation, muni de la topologie des homéomorphismes locaux.

Le foncteur $\theta: \operatorname{Sm}(\mathbf{C}) \rightarrow C W$ qui à un schéma $X$ associe la variété $X(\mathbf{C})$ des points complexes est continu. Il induit un foncteur $\theta^{s}: \widetilde{\mathrm{Sm} \mathbf{C}} \rightarrow \widetilde{C W}$ prolongeant $\theta$.

Proposition 3.1.1. Si $X$ est un schéma projectif lisse, l'image $\theta^{s}\left(\mathbf{Z}_{t r}(X)\right)$ est le faisceau

$$
U \mapsto \operatorname{Hom}\left(U, \coprod_{d \geq 0} S^{d} X(\mathbf{C})\right)^{+}
$$

où le schéma $S^{d} X$ est la puissance symétrique du schéma $X$ et pour tout monoïde $M$, on désigne par $M^{+}$le groupe groupe de Grothendieck associé.

Demonstration : le résultat repose essentiellement sur le théorème de Suslin et Voevodsky.

Theoreme 3.1.2. (Suslin et Voedvosky [SV96 Theorem 6.8])

Si $X$ est un schéma quasi-projectif sur un corps $k$, on a, pour tout schéma (normal et connexe) $S$, un isomorphisme de groupes

$$
\mathbf{Z}_{t r}(X)(S)=\operatorname{Hom}_{\operatorname{Sch} k}\left(S, \coprod_{d \geq 0} S^{d}(X)\right)^{+},
$$

où la catégorie Sch $k$ est celle des schémas de type fini sur $k$.

Le foncteur $\theta^{s}$ respectant les foncteurs représentables, il envoie, pour tout entier positif $d$ et tout schéma lisse $X$, le faisceau représenté par $X^{d}$ sur le faisceau représenté par $X^{d}(\mathbf{C})$. Par ailleurs, le foncteur $\theta^{s}$ commute aux colimites finies (comme le quotient par les groupes de permutation) mais également aux colimites quelconques ([SGA4] III 1.3) : il envoie le faisceau représenté par 
le ind-schéma lisse $\coprod_{d \geq 0} S^{d} X$ sur le faisceau représenté par l'espace $\coprod_{d \geq 0} S^{d}(X(\mathbf{C}))$. Comme le foncteur est compatible aux structures algébriques, la proposition (3.1.1.) s'en déduit.

Composant le foncteur $\theta^{s}$ avec le foncteur exact oubli de transferts, nous obtenons un foncteur exact à droite qui se factorise dans les faisceaux abéliens en un foncteur monoïdal

$$
\Phi: \operatorname{Shv}_{N i s}(\operatorname{Smcor} \mathbf{C}) \rightarrow \widetilde{C W}_{A b}
$$

de la catégorie des faisceaux de Nisnevich avec transferts vers le topos abélien $\widetilde{C W}_{A b}$. En composant de plus avec le foncteur complexe de Suslin $C_{*}$ qui est exact, nous obtenons un foncteur, toujours exact à droite,

$$
\Psi: \operatorname{Shv}_{N i s}(\operatorname{Smcor} \mathbf{C}) \rightarrow C^{-}\left(\widetilde{C W}_{A b}\right)
$$

où $C^{-}\left(\widetilde{C W}_{A b}\right)$ est la catégorie abélienne des complexes bornés supérieurement de faisceaux abéliens. C'est ce foncteur que nous dérivons en utilisant la classe $\Sigma$ des sommes de faisceaux $\oplus_{\alpha} \mathbf{Z}_{t r}\left(V_{\alpha}\right)$ où les $V_{\alpha}$ parcourent les schémas lisses quasi-projectifs tels que la variété des points complexes $V_{\alpha}(\mathbf{C})$ soit à composantes connexes contractiles. Cette classe $\Sigma$ d'objets est bien sûr stable par somme directe et permet via la proposition 1.3.1.1 de construire des résolutions à gauche de tout faisceau avec transferts. Pour que le foncteur $\Psi$ admette un foncteur dérivé à gauche, il suffit par [Ver77] de vérifier que les objets de $\Sigma$ sont acycliques à gauche en montrant

Lemme 3.1.3. Soit $N$ un complexe acyclique et borné supérieurement d'objets $\oplus_{\alpha} \mathbf{Z}_{t r}\left(V_{\alpha}\right)$ de $\Sigma$. Alors $\Psi(N)$ est acyclique.

Demonstration : pour tout entier $i$, nous devons montrer que le faisceau de Nisnevich associé au préfaisceau $H_{i}(\Psi(N))$ est nul. Cela revient à montrer que pour tout préfaisceau $F$ tel que le faisceau associé $F_{N i s}$ soit nul, les dérivés $L^{n} \Phi(F)$ sont nuls. Comme en [MVW] 8.15 on se ramène au cas d'un complexe de recouvrement de Cech et nous devons montrer le lemme suivant :

Lemme 3.1.4. Si $\mathcal{U}=\left\{U_{i} \rightarrow X\right\}$ est un recouvrement de Nisnevich d'un schéma $X$ lisse quasiprojectif sur $\mathbf{C}$ par des ouverts dont les points complexes sont à composantes contractiles, alors l'image par le foncteur $\Phi$ du complexe de Cech $\check{N}(\mathcal{U} / X)$ est acyclique.

Ce lemme repose sur les travaux de Suslin et Voevodsky en [SV96] section 10 qui ont réinterprété le théorème de Dold-Thom en

Proposition 3.1.5. Si $X$ est un schéma lisse quasi-projectif sur $\mathbf{C}$, l'image $\Phi\left(\mathbf{Z}_{t r}(X)\right)$ est quasiisomorphe au complexe des chaînes singulières de la variété topologique $X(\mathbf{C})$.

Demonstration : par dualité il est équivalent de montrer que le bicomplexe de cochaînes

$$
\operatorname{Sing} \cdot(X(\mathbf{C})) \rightarrow \operatorname{Sing} \cdot(\check{N} \mathcal{U}(\mathbf{C}))
$$

est acyclique. D'après la proposition 3.1.5 le bicomplexe $\operatorname{Sing}^{\prime}\left(\check{N}_{\mathcal{U}}(\mathbf{C})\right)$ calcule la cohomologie de Cech $\breve{H}^{*}(X(\mathbf{C}))$ de $X(\mathbf{C})$ qui est égale à la cohomologie singulière de $\left.X(\mathbf{C})\right)$ par un théorème de Cartan ([Go58], 5.9.2).

Nous en déduisons un foncteur $L \Psi: D^{-}\left(\operatorname{Shv}_{N i s}(\operatorname{Smcor} \mathbf{C})\right) \rightarrow D(A b)$ qui par le lemme d'homotopie (1.3.3.2) se factorise en un foncteur dit de réalisation topologique $t_{\mathbf{C}}: \mathbf{D M}^{-, \text {eff }}(\mathbf{C}) \rightarrow D(\mathcal{A} b)$.

Par le théorème d'Eilenberg-Zilber, le foncteur $\Psi$ est compatible au produit et son dérivé $t_{\mathbf{C}}$ commute au produit de $\mathbf{D} \mathbf{M}^{-, \text {eff }}(\mathbf{C})$ également défini à partir des résolutions par les $\mathbf{Z}_{t r}(X)$.

L'image de $\mathbf{Z}(1)=C_{*} \mathbf{Z}_{t r}\left(\mathbf{G}_{m}^{\wedge 1}\right)[-1]$ est le complexe calculant l'homologie singulière réduite de $\mathbf{C}^{*}$, décalé de -1 , d'où $t_{\mathbf{C}}(\mathbf{Z}(1)) \simeq 2 i \pi \mathbf{Z}$, complexe concentré en degré 0 , par le théorème des résidus. Comme le foncteur $t_{\mathbf{C}}$ est tensoriel et envoie le motif de Tate $\mathbf{Z}(1)$ sur un objet inversible, il s'étend en un foncteur de $\mathbf{D} \mathbf{M}^{-}(\mathbf{C})$ et les résultats de ce paragraphe se résument en

Theoreme 3.1.6. Il existe un foncteur tensoriel de réalisation topologique

$$
\begin{array}{ccc}
t_{\mathbf{C}}: \mathbf{D M}^{-}(\mathbf{C}) & \rightarrow D(\mathcal{A} b) \\
\mathbf{M} & \mapsto \mathbf{M}(\mathbf{C}),
\end{array}
$$


qui pour le motif $\mathbf{M}=\mathbf{M}(X)$ associé à un schéma $X$ quasi-projectif lisse sur $\mathbf{C}$ permet de représenter la cohomologie singulière de $X(\mathbf{C})$, c'est-à-dire

$$
H^{p}(X(\mathbf{C}), \mathbf{Z})=\operatorname{Hom}_{D^{-}(\mathcal{A} b)}\left(t_{\mathbf{C}}(\mathbf{M}(X)), \mathbf{Z}[p]\right) .
$$

La compatibilité du foncteur de réalisation au produit impose

$$
t_{\mathbf{C}}(\mathbf{Z}(q))=(2 i \pi)^{q} \mathbf{Z}
$$

Comme, pour les schémas algébriques sur $\mathbf{C}$, la topologie analytique est intermédiaire entre la topologie de Nisnevich et la topologie usuelle, le foncteur $t_{\mathbf{C}}$ se factorise à travers $t_{a n}: \mathbf{D M}^{-}(\mathbf{C}) \rightarrow$ $\mathbf{D M}_{a n}^{-}$. Par le lemme de Yoneda, on a pour tout schéma lisse $X$ et tout faisceau analytique avec transferts $F$, un isomorphisme

$$
\operatorname{Hom}_{\mathbf{D M}_{a n}^{-}}\left(\mathbf{M}(X)_{a n}, F\right)=H_{a n}(X(\mathbf{C}), F)
$$

en particulier pour le faisceau constant $\mathbf{Z}$

$$
\operatorname{Hom}_{\mathbf{D M}_{a n}^{-}}\left(\mathbf{M}(X)_{a n}, \mathbf{Z}\right)=H_{\text {sing }}(X(\mathbf{C}), \mathbf{Z}) .
$$

On en déduit, puisque les motifs de schémas lisses engendrent $\mathbf{D M}_{\mathrm{gm}}(\mathbf{C})$

Proposition 3.1.7. On a $t_{a n}(\mathbf{Z}(q))=(2 i \pi)^{q} \mathbf{Z}$ et la restriction de $t_{a n}$ à $\mathbf{D M}_{\mathrm{gm}}(\mathbf{C})$ induit des isomorphismes

$$
\operatorname{Hom}_{D^{-}(\mathcal{A} b)}\left(t_{\mathbf{C}}(\mathbf{M}),(2 i \pi)^{q} \mathbf{Z}\right) \simeq \operatorname{Hom}_{\mathbf{D M}_{a n}^{-}}\left(\mathbf{M}(X)_{a n}, \mathbf{Z}(q)_{a n}\right)
$$

pour tout motif géométrique $\mathbf{M}$ sur $\mathbf{C}$.

\subsection{Réalisation topologique des motifs sur R.}

Si le schéma $X$ est défini sur $\mathbf{R}$, la variété analytique $X(\mathbf{C})=\left(X \times_{\text {Spec }} \mathbf{R}\right.$ Spec $\left.\mathbf{C}\right)(\mathbf{C})$ est munie d'une action continue de la conjugaison complexe $F_{\infty}$. En suivant cette action dans la construction précédente, on montre que le foncteur de réalisation topologique se factorise en un diagramme

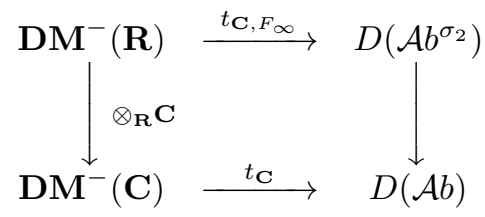

où $\mathcal{A} b^{\sigma_{2}}$ est la catégorie abélienne des groupes abéliens munis d'une involution et la flèche de droite est induite par l'oubli de l'involution.

Le motif de Tate $\mathbf{Z}(1)$ est réel et, sur sa réalisation $t_{\mathbf{C}}(\mathbf{Z}(1))$, l'involution est induite par le changement d'orientation de $\mathbf{S}^{1}$ dans $\mathbf{C}^{*}$ et agit par multiplication par -1 . 


\subsection{Réalisation de Betti des motifs}

Pour tout plongement $\sigma: k \rightarrow \mathbf{C}$, l'extension des scalaires $\sigma_{\mathbf{C}}: \mathbf{D M}^{-}(k) \rightarrow \mathbf{D M}^{-}(\mathbf{C})$ construite en 1.4, composée avec la réalisation topologique, définit un foncteur

$$
\begin{array}{ccc}
t_{\sigma}: \mathbf{D M}^{-}(k) & \rightarrow & D(\mathcal{A} b) \\
\mathbf{M} & \mapsto \mathbf{M}_{\sigma}(\mathbf{C}):=t_{\mathbf{C}} \circ \sigma_{\mathbf{C}}(\mathbf{M}) .
\end{array}
$$

Si le plongement $\sigma$ est réel, le foncteur $t_{\sigma}$ se factorise dans la catégorie des groupes abéliens munis d'une involution (3.2).

On pose alors $\mathbf{H}_{\sigma}(\mathbf{M}, q)=\operatorname{Hom}_{D(\mathcal{A} b)}\left(\mathbf{M}_{\sigma}(\mathbf{C}),(2 i \pi)^{q} \mathbf{Z}\right)$ et l'on définit

Definition 3.3.1. Pour tout complexe motivique $\mathbf{M}$ de $\mathbf{D M}^{-}(k)$, tout plongement $\sigma: k \rightarrow \mathbf{C}$ et tout entier $q \geq 0$, la réalisation entière (resp. réalisation) de $\operatorname{Betti} \mathbf{H}_{\sigma}^{*}(, \mathbf{Z}(q))\left(\operatorname{resp} . \mathbf{H}_{\sigma}^{*}(, q)\right.$ ) est le groupe abélien (resp. Q-espace vectoriel) gradué sur $\mathbf{Z}$

$$
\mathbf{H}_{\sigma}(\mathbf{M}, \mathbf{Z}(q))=\oplus_{p \in \mathbf{Z}} \mathbf{H}_{\sigma}^{p}(\mathbf{M}, \mathbf{Z}(q)),
$$

et

$$
\mathbf{H}_{\sigma}^{p}(\mathbf{M}, q)=\mathbf{Q} \otimes \mathbf{z} \mathbf{H}_{\sigma}^{p}(\mathbf{M}, \mathbf{Z}(q)) .
$$

Si le plongement $\sigma$ est réel, le complexe $\mathbf{M}_{\sigma}(\mathbf{C})$ est muni d'une involution induite par la conjugaison complexe et les réalisations $\mathbf{H}_{\sigma}^{*}(, \mathbf{Z}(q))$ et $\mathbf{H}_{\sigma}^{*}(, q)$ héritent de cette structure.

Le foncteur $t_{\sigma}$ induit directement des classes de Chern, pour des entiers $p$ et $q$ et tout complexe motivique $\mathbf{M}$ de $\mathbf{D} \mathbf{M}^{-}(k)$

$$
c_{\sigma}^{p, q}: H^{p, q}(\mathbf{M})=\operatorname{Hom}_{\mathbf{D M}^{-}(k)}(\mathbf{M}, \mathbf{Z}(q)[p]) \rightarrow \operatorname{Hom}_{D(\mathcal{A} b)}\left(\mathbf{M}_{\sigma}(\mathbf{C}),(2 i \pi)^{q} \mathbf{Z}[p]\right)=\mathbf{H}_{\sigma}^{p}(\mathbf{M}, \mathbf{Z}(q)) .
$$

Si le complexe motivique $\mathbf{M}=\mathbf{M}(X)$ est le motif d'un schéma quasi-projectif lisse sur $k$, les groupes de réalisation de Betti coïncident d'après (3.1.5) avec les groupes de cohomologie singulière

$$
\mathbf{H}_{\sigma}^{p}(\mathbf{M}(X), \mathbf{Z}(q))=H^{p}\left(X(\mathbf{C}),(2 i \pi)^{q} \mathbf{Z}\right) .
$$

3.3.2. Filtration par le poids

Par ailleurs, la réalisation de $\operatorname{Betti} \mathbf{H}_{\sigma}(, \mathbf{Z})$, pour $q=0$, étant un foncteur cohomologique, elle hérite de la filtration par le poids décrite en (1.5.9), à savoir, pour $i \in \mathbf{Z}$,

$$
W_{i} \mathbf{H}_{\sigma}(\mathbf{M}, \mathbf{Z})=\operatorname{Im}\left(\mathbf{H}_{\sigma}\left(w_{\leq i} \mathbf{M}, \mathbf{Z}\right) \rightarrow \mathbf{H}_{\sigma}(\mathbf{M}, \mathbf{Z})\right) .
$$

Le calcul fait en (2.2) dans le cadre de la réalisation de De Rham montre que cette filtration coïncide avec celle de Deligne après avoir tensorisé par $\mathbf{C}$. En particulier, pour un schéma lisse $X$ que l'on plonge dans $\bar{X}$, la suite spectrale de Bondarko associé à $\mathbf{H}_{\sigma}$ est isomorphe (à renumérotation près) à la suite spectrale de Leray de la cohomologie singulière pour l'inclusion $X(\mathbf{C}) \hookrightarrow \bar{X}(\mathbf{C})$.

Definition 3.3.3. Pour tout $q \in \mathbf{Z}$ et tout motif géométrique $\mathbf{M}$, on appelle filtration par le poids de la réalisation de Betti $\mathbf{H}_{\sigma}(\mathbf{M}, \mathbf{Z}(q))$, la filtration croissante indexée par $\mathbf{Z}$ déduite par la filtration par le poids de Bondarko

$$
W_{i} \mathbf{H}_{\sigma}(\mathbf{M}, \mathbf{Z}(q))=\operatorname{Im}\left(\mathbf{H}_{\sigma}\left(w_{\leq i+2 q} \mathbf{M}, \mathbf{Z}(q)\right) \rightarrow \mathbf{H}_{\sigma}(\mathbf{M}, \mathbf{Z}(q))\right) .
$$

Le principe (0.2) se traduit en

Proposition 3.3.4. Pour chaque plongement $\sigma$, et chaque entier positif $q$ les foncteurs réalisations de Betti $\mathbf{H}_{\sigma}(, \mathbf{Z}(q))$ induisent des foncteurs cohomologiques de la catégorie des motifs géométriques sur $k$ vers la catégorie des $\mathbf{Z}$-modules filtrés de type fini. Si de plus le plongement est réel, la réalisation est munie d'une involution induite par la conjugaison complexe.

Comme dans le cas de la réalisation de De Rham, nous avons

Proposition 3.3.5. Soient $\mathbf{M}$ un motif géométrique effectif, $\mathbf{M}^{(\cdot)}$ un complexe des poids associé à $\mathbf{M}$. La cohomologie du complexe total $\mathbf{K}_{\sigma}$ du bicomplexe $\mathrm{R} \cdot \operatorname{Hom}\left(t_{\sigma}\left(\mathbf{M}^{(\cdot)}\right), \mathbf{Z}(q)\right)$ calcule la réalisation de Betti de $\mathbf{M}$. 


\section{Réalisation de Hodge}

\subsection{Comparaison des réalisations de De Rham et de Betti}

Fixons un plongement $\sigma: k \rightarrow \mathbf{C}$. Pour comparer $\operatorname{Hom}_{D(\mathcal{A} b)}\left(\mathbf{M}_{\sigma}(\mathbf{C}), \mathbf{Z}(q)\right)$ et $\operatorname{Hom}_{\mathbf{D M}^{-}(k)}(\mathbf{M}, \boldsymbol{\Omega})$ pour tout motif $\mathbf{M}$, nous partons des classes de Chern (2.1.5) $\mathbf{Z}(q) \rightarrow \boldsymbol{\Omega}$ et appliquons le foncteur de réalisation topologique $t_{\sigma}(3.3)$ qui induit les flèches

$$
(2 i \pi)^{q} \mathbf{Z} \rightarrow t_{\sigma}(\boldsymbol{\Omega})
$$

dans la catégorie des complexes de groupes abéliens. En tensorisant avec le corps des nombres complexes, nous obtenons

Lemme 4.1.1. Le morphisme $\mathbf{C} \rightarrow t_{\sigma}(\boldsymbol{\Omega})$ est un quasi-isomorphisme de complexes de groupes abéliens.

Demonstration : au niveau des faisceaux, le foncteur de réalisation topologique se factorise à travers le foncteur $t_{a n}$ de (1.3.2.1). Le lemme de Poincaré implique que dans la catégorie dérivée des faisceaux anaytiques, le complexe des différentielles holomorphes $\boldsymbol{\Omega}_{a n}$ est une résolution du faisceau constant $\mathbf{C}$. Il induit un isomorphisme $\mathbf{C} \simeq \boldsymbol{\Omega}_{a n}$ dans $\mathbf{D} \mathbf{M}_{a n}$ qui se transporte dans $D(\widetilde{C W})$ puis $D(\mathcal{A} b)$.

On en déduit, pour tout complexe motivique $\mathbf{M}$, une flèche

$$
\mathbf{H}_{D R}(\mathbf{M}) \otimes_{k} \mathbf{C} \rightarrow \mathbf{H}_{\sigma}^{\cdot}(\mathbf{M}, 0) \otimes_{\mathbf{Q}} \mathbf{C}
$$

Par le théorème de De Rham, cette flèche est un isomorphisme d'espaces vectoriels gradués de dimension finie pour tout motif $\mathbf{M}(X)$ d'un schéma lisse projectif $X$, isomorphisme compatible par construction avec l'action de la conjugaison complexe si le plongement $\sigma$ est réel. Comme nous l'avons déjà remarqué, les filtrations par le poids coïncident (cf 3.3.2). Les motifs $\mathbf{M}(X)$ engendrant la catégorie des motifs géométriques, on a

Proposition 4.1.2. Pour tout motif géométrique $\mathbf{M}$ de $\mathbf{D M}_{\mathrm{gm}}$, tout entier $q$ positif et tout entier $p$, on a un isomorphisme de $\mathbf{C}$-espaces vectoriels filtrés (par la filtration par le poids) de dimension finie

$$
\mathbf{H}_{D R}^{p}(\mathbf{M}) \otimes_{k} \mathbf{C} \simeq \mathbf{H}_{\sigma}^{p}(\mathbf{M}, 0) \otimes_{\mathbf{Q}} \mathbf{C}
$$

compatible avec l'action de la conjugaison complexe si le plongement est réel. 


\subsection{Théorie de Hodge}

Une structure de Hodge mixte sur un sous-corps $k$ de $\mathbf{C}$ est la donnée d'un $\mathbf{Z}$-module de type fini $H_{\mathbf{Z}}$, d'une filtration croissante $W_{n}$ sur $H_{\mathbf{Q}}=\mathbf{Q} \otimes_{\mathbf{z}} H_{\mathbf{Z}}$, d'une filtration décroissante $F^{p}$ sur $H_{k}=k \otimes_{\mathbf{Z}} H_{\mathbf{Z}}$, tels que le système des trois filtrations $(W, F, \bar{F})$ qui en découle sur $H_{\mathbf{C}}=\mathbf{C} \otimes_{\mathbf{Z}} H_{\mathbf{Z}}$ soit un système de trois filtrations opposées [D71](1.2.13). La catégorie des structures de Hodge mixtes est une catégorie abélienne (loc. cit. 2.3.5).

La filtration par le poids de Bondarko sur $\mathbf{H}_{\sigma}^{p}(\mathbf{M}, \mathbf{Z})$, la filtration de Hodge sur $\mathbf{H}_{D R}^{p}(\mathbf{M})$ et l'isomorphisme de comparaison De Rham-Betti permettent d'associer à un complexe motivique, objet de $\mathbf{D M}_{\mathrm{gm}}(k)$, une structure de Hodge mixte sur $k$. Le théorème 0.1 se traduit en

Theoreme 4.2.1. Pour chaque plongement $\sigma: k \hookrightarrow \mathbf{C}$, la donnée du $\mathbf{Z}$-module $\mathbf{H}_{\sigma}(\mathbf{M}, \mathbf{Z})$, de la filtration $W$ par le poids de Bondarko sur $\mathbf{Q} \otimes \mathbf{z} \mathbf{H}_{\sigma}(\mathbf{M}, \mathbf{Z})$, du $k$-espace vectoriel filtré par la filtration de Hodge $\left(\mathbf{H}_{D R}(\mathbf{M}), F^{\cdot}\right)$, du complexifié $\mathbf{H}_{\mathbf{C}}(\mathbf{M})=\mathbf{C} \otimes \mathbf{H}_{D R}(\mathbf{M})$, muni des trois filtrations $W$., $F$ et de sa complexe conjuguée $\bar{F}^{*}$, pour tout motif géométrique $\mathbf{M}$, induit un foncteur cohomologique de la catégorie $\mathbf{D M}_{\mathrm{gm}}(k)$ des motifs géométriques vers la catégorie des structures de Hodge mixtes sur $k$.

Demonstration :

Il suffit de traiter le cas où $\mathbf{M}$ est un motif géométrique effectif. La démonstration se fait en deux temps.

Lemme 4.2.2. Si M est un motif de Chow, les données du théorème fournissent une structure de Hodge pure.

Demonstration : Si $\mathbf{M}$ est le motif d'un schéma lisse projectif $X$, c'est un résultat classique, puisque les réalisations de $\mathbf{M}$ coïncident avec les réalisations classiques de $X$.

Si M est une somme finie ou un facteur direct d'un $\mathbf{M}(X)$, pour $X$ projectif lisse, cela provient du théorème de Deligne que la catégorie des structures de Hodge est abélienne (cf. [D71]).

Lemme 4.2.3. La donnée des bicomplexes

a) $K_{B}^{p, q}=\mathrm{R}^{q} \operatorname{Hom}\left(\mathbf{M}_{\sigma}^{(-p)}(\mathbf{C}), \mathbf{Z}\right)$;

b) $K_{\mathbf{Q}}^{p, q}=\mathrm{R}^{q} \operatorname{Hom}\left(\mathbf{M}_{\sigma}^{(-p)}(\mathbf{C}), \mathbf{Q}\right)$, muni de la filtration par les poids;

c) $K_{D R}^{p, q}=\mathrm{R}^{q} \operatorname{Hom}\left(\mathbf{M}^{(-p)}, \boldsymbol{\Omega}\right)$, muni de la première filtration et de la filtration de Hodge, est un Z-complexe de Hodge mixte DG au sens de Deligne ([D74], 8.1.10).

Demonstration : il suffit de vérifier que pour chaque $p, K^{p, \cdot}$ est un complexe de Hodge mixte, ce qui provient du fait que chaque $M^{(-p)}$ est un motif de Chow et du lemme précédent, qui permet de préciser que pour $p$ fixé, $K^{p, \cdot}$ est un complexe de Hodge pur de poids 0 .

Le théorème de Deligne (8.1.15 de [D74]) permet de conclure que pour chaque motif géométrique $\mathbf{M}$, les données du théorème, qui sont les cohomologies des données du lemme, définissent une structure de Hodge mixte. On note que le complexe total associé au bicomplexe $K^{p, q}$, muni de sa filtration diagonale est bien le complexe $\mathbf{K}_{D R}$ muni de la filtration par le poids de Bondarko.

Sachant que la filtration par le poids est indépendante des choix ([Bo10]), il nous faut vérifier

Proposition 4.2.4. Si $\mathbf{M}$ est un motif géométrique, la filtration de Hodge sur $\mathbf{H}_{D R}(\mathbf{M})$ est indépendante du choix du complexe des poids.

Demonstration : Soient $\mathbf{M}^{(\cdot)}$ et $\mathbf{M}^{(\cdot)}$ deux complexes de poids du motif $\mathbf{M}$ que l'on peut supposer effectif. Bondarko a montré en [Bo09] que les deux complexes étaient homotopes. Les morphismes et les homotopies étant définis au niveau des motifs, ils induisent sur $\mathbf{H}_{D R}(\mathbf{M})$ des morphismes compatibles aux filtrations de Hodge définies par troncature. Ainsi les deux complexes $\mathbf{M}^{(\cdot)}$ et $\mathbf{M}^{\prime(\cdot)}$ définissent sur $\mathbf{H}_{\mathbf{C}}(\mathbf{M})$ deux structures de Hodge mixtes et l'équivalence d'homotopie entre les deux complexes un automorphisme de $\mathbf{H}_{\mathbf{C}}(\mathbf{M})$ compatible à toutes les filtrations : d'après [D71] cet automorphisme induit un isomorphisme de structures de Hodge mixtes.

On a ainsi construit un foncteur cohomologique $\mathbf{H}_{\text {Hodge }}$ de la catégorie $\mathbf{D} \mathbf{M}_{\mathrm{gm}}^{\mathrm{eff}}(k)$ dans la catégorie des structures de Hodge mixtes. Pour conclure la démonstration du théorème, il suffit de préciser que le foncteur est multiplicatif et que les structures de Hodge des motifs de Tate sont inversibles, en rappelant 
Proposition 4.2.5. La réalisation de Hodge des motifs de Tate $\mathbf{Z}(n)$ est la structure de Hodge pure de poids $2 n$ suivante

$$
\begin{aligned}
\mathbf{H}_{\text {Hodge }}^{i}(\mathbf{Z}(n)) & =0 \quad \text { si } i \neq 0, \\
\mathbf{H}_{\text {Hodge }}^{0}(\mathbf{Z}(n)) & =\left(\mathbf{H}_{\sigma}^{0}(\mathbf{Z}(n)), \mathbf{H}_{D R}^{0}(\mathbf{Z}(n)), \mathbf{C} \otimes \mathbf{H}_{D R}^{0}(\mathbf{Z}(n)), W^{\prime}, F^{\cdot}, \bar{F}^{\cdot}\right) \\
& =\left(\mathbf{Z}, k, \mathbf{C}, W . F^{\cdot}, \bar{F}^{\cdot}\right)
\end{aligned}
$$

avec pour filtration par le poids

$$
\begin{array}{ll}
W_{i} \mathbf{H}_{\sigma}^{0}(\mathbf{Z}(n))=0 & \text { pour } i \leq 2 n-1 \\
W_{i} \mathbf{H}_{\sigma}^{0}(\mathbf{Z}(n))=k & \text { pour } i \geq 2 n
\end{array}
$$

et pour filtration de Hodge

$$
\begin{array}{ll}
F^{p} \mathbf{H}_{D R}^{0}(\mathbf{Z}(n))=0 & \text { pour } p \geq n+1 \\
F^{p} \mathbf{H}_{D R}^{0}(\mathbf{Z}(n))=k & \text { pour } p \leq n .
\end{array}
$$

Demonstration : cela provient du triangle exact scindé [MVW Chap.15]

$$
\mathbf{M}\left(\mathbf{P}^{n-1}\right) \rightarrow \mathbf{M}\left(\mathbf{P}^{n}\right) \rightarrow \mathbf{Z}(n)[2 n] \rightarrow \mathbf{M}\left(\mathbf{P}^{n-1}\right)[1]
$$

et du fait que l'espace projectif $\mathbf{P}^{n}$ a comme nombres de Hodge non nuls $h^{p, p}$ pour $0 \leq p \leq n$. Remarque 4.2.6. Remarquons que pour tout $q \in \mathbf{Z}$ et tout motif géométrique $\mathbf{M}$,

$$
\mathbf{H}_{\sigma}(\mathbf{M}, \mathbf{Z}(q))=\mathbf{H}_{\sigma}(\mathbf{M}(-q), \mathbf{Z})
$$

et que cet isomorphisme est compatible avec la filtration par les poids décrite en 3.3.3. On peut ainsi définir une réalisation de Hodge pour tout $q \in \mathbf{Z}$, en posant

$$
\mathbf{H}_{\text {Hodge }}(\mathbf{M}, q)=\mathbf{H}_{\text {Hodge }}(\mathbf{M}(-q)) .
$$

Proposition 4.2.7. Si $\mathbf{M}=\mathbf{M}(X)$ est le motif d'un schéma $X$, alors la structure de Hodge mixte définie en 4.2.1 coïncide avec celle définie par Deligne en [D74].

Demonstration : si le schéma $X$ est lisse et projectif, c'est vrai par construction (2.3.5). Si le schéma $X$ est lisse, on le plonge dans un schéma projectif lisse $\bar{X}$ tel que le schéma complémentaire $Y=\bar{X}-X$ soit un diviseur à croisements normaux à composantes $Y_{n}$ lisses, où $n \leq N$. On choisit comme complexe des poids de $\mathbf{M}(X)$ le complexe défini en (1.5.4), avec les notations de [D71]

$$
\mathbf{M}(X)^{(i)}=\left\{\begin{array}{lc}
\mathbf{M}\left(\widetilde{Y^{i}}\right)(i)[2 i] & \text { si } 0 \leq i \leq N \\
0 & \text { sinon }
\end{array}\right.
$$

Les morphismes propres $i_{m}: \widetilde{Y^{m}} \rightarrow \bar{X}$ permettent de définir un bicomplexe de $\mathcal{O}_{\bar{X}}$-modules $i_{m \star} \Omega_{\widetilde{Y}^{m}}^{p}$ et un complexe de $\mathcal{O}_{\bar{X}}$-algèbres différentielles graduées

$$
\oplus_{1 \leq m \leq N} i_{m \star} \Omega_{\widetilde{Y}^{m}}^{-{ }^{-m}} .
$$

En [D71] Deligne définit la structure de Hodge mixte pour $X$ à partir du complexe de De Rham à pôles logarithmiques $\Omega_{\bar{X}}\langle Y\rangle$ et construit une application (loc. cit. 3.1.5.2)

$$
\Omega_{\bar{X}}\langle Y\rangle \rightarrow \oplus_{1 \leq m \leq N} i_{m \star} \Omega_{\widetilde{Y}^{m}}^{\cdot-m}\left(\epsilon^{m}\right)[-m]
$$

qui induit un morphisme de bicomplexes

$$
\begin{aligned}
\mathrm{R} \operatorname{Hom}\left(\bar{X}, \Omega_{\bar{X}}^{\prime}\langle Y\rangle\right) \longrightarrow & \oplus_{1 \leq m \leq N} \mathrm{R} \operatorname{Hom}\left(\bar{X}, i_{m \star} \Omega_{\widetilde{Y}^{m}}\left(\epsilon^{m}\right)[-2 m]\right. \\
& =\oplus_{1 \leq m \leq N} \mathrm{R} \operatorname{Hom}\left(\widetilde{Y}^{m}, \Omega_{\widetilde{Y}^{m}}\left(\epsilon^{m}\right)[-2 m]\right) \\
& =\oplus_{1 \leq m \leq N} \mathrm{R} \operatorname{Hom}\left(\mathbf{M}(X)^{(m)}, \boldsymbol{\Omega}\right)
\end{aligned}
$$


compatible aux filtrations par le poids et de Hodge.

Ce morphisme induit un isormophisme via le résidu de Poincaré sur les termes $\mathrm{Gr}_{W}$ et donc un isomorphisme entre les structures de Hodge respectives.

Si le schéma $X$ est singulier, Deligne construit un schéma simplicial lisse à partir d'éclatements, puis un Z-complexe de Hodge mixte DG. On vérifie que par les triangles d'éclatements (1.3.5.4) on peut choisir un complexe des poids de telle sorte que le $\mathbf{Z}$-complexe de Hodge mixte DG construit en (4.2.3) soit quasi-isomorphe à celui de Deligne. On peut également utiliser le raisonnement de Huber (cf 4.4 ci-dessous).

\subsubsection{Application}

La théorie de Hodge des schémas projectifs lisses complexes $X$ permet d'identifier $F^{p} \mathbb{H} \cdot\left(X, \Omega_{X}\right)$ à $\mathbb{H}^{\cdot}\left(X, \sigma \geq p \Omega_{X}\right)$ et le conoyau $\mathbb{H}^{\cdot}\left(X, \Omega_{X}\right) / F^{p} \mathbb{H}^{\cdot}\left(X, \Omega_{X}\right)$ à $\mathbb{H}^{\prime}\left(X, \Omega_{X}^{<p}\right)$. Cette propriété s'étend aux motifs sur C. Considérons le complexe $\boldsymbol{\Omega}^{<p}$ de $D^{-}\left(\operatorname{Shv}_{N i s}(\operatorname{Smcor}(\mathbf{C}))\right)$

$$
0 \rightarrow \mathcal{O} \rightarrow \boldsymbol{\Omega}^{1} \rightarrow \cdots \rightarrow \boldsymbol{\Omega}^{p-1} \rightarrow 0
$$

Lemme 4.2.8.1. Pour tout entier $p \geq 0$, pour tout motif géométrique effectif de $\mathbf{D M}^{-, \text {eff }}(\mathbf{C})$ et tout complexe des poids $\mathbf{M}^{(\cdot)}$ de $\mathbf{M}$, l'hypercohomologie du bicomplexe

$$
\left.\mathrm{R}^{\cdot} \operatorname{Hom}_{D\left(\operatorname{Shv}_{N i s}(\operatorname{Smcor}(\mathbf{C}))\right)}\left(\mathbf{M}^{(\cdot)}, \mathbf{\Omega}^{<p}\right)\right)
$$

s'identifie au conoyau

$$
\mathbf{H}_{D R}^{\cdot}(\mathbf{M}) / F^{p} \mathbf{H}_{D R}^{\cdot}(\mathbf{M}) .
$$

Demonstration : on plonge $\mathbf{D} \mathbf{M}^{-, \text {eff }}(\mathbf{C})$ dans $D\left(\operatorname{Shv}_{N i s}(\operatorname{Smcor}(\mathbf{C}))\right)$ où l'on a, avec les notations évidentes, le triangle distingué

$$
0 \rightarrow \sigma \geq p \boldsymbol{\Omega} \rightarrow \boldsymbol{\Omega} \rightarrow \boldsymbol{\Omega}^{<p} \rightarrow 0
$$

On déduit du cas des schémas projectifs lisses que le triangle (4.2.8.2) induit pour tout motif $\mathbf{M}$ de poids 0 une suite exacte courte

$$
0 \rightarrow \operatorname{Hom}_{D\left(\operatorname{Shv}_{N i s}(\operatorname{Smcor}(\mathbf{C}))\right)}(\mathbf{M}, \sigma \geq p) \rightarrow \mathbf{H}_{D R}(\mathbf{M}) \rightarrow \operatorname{Hom}_{D^{-}\left(\operatorname{Shv}_{N i s}(\operatorname{Smcor}(\mathbf{C}))\right)}\left(\mathbf{M}, \boldsymbol{\Omega}^{<p}\right) \rightarrow 0
$$

avec $\operatorname{Hom}_{D\left(\operatorname{Shv}_{N i s}(\operatorname{Smcor}(\mathbf{C}))\right)}(\mathbf{M}, \sigma \geq p \boldsymbol{\Omega})=F^{p} \mathbf{H}_{D R}(\mathbf{M})$. Le lemme s'ensuit par une récurrence sur la longueur du complexe de poids de $\mathbf{M}$.

Remarque : le lemme signifie que bien que $\boldsymbol{\Omega}^{<p}$ ne soit pas un complexe motivique on peut donner un sens à $\mathrm{R} \cdot \operatorname{Hom}\left(\mathbf{M}, \boldsymbol{\Omega}^{<p}\right)$ en posant

$$
\mathrm{R}^{\cdot} \operatorname{Hom}\left(\mathbf{M}, \boldsymbol{\Omega}^{<p}\right)=\text { Tot } \quad \operatorname{cône}\left(F^{p} \mathrm{R}^{\cdot} \operatorname{Hom}_{\mathbf{D M}(\mathbf{C})}\left(\mathbf{M}^{(\cdot)}, \boldsymbol{\Omega}\right) \rightarrow \mathrm{R}^{\cdot} \operatorname{Hom}_{\mathbf{D M}(\mathbf{C})}\left(\mathbf{M}^{(\cdot)}, \boldsymbol{\Omega}\right)\right)
$$

qui est bien défini à homotopie près.

4.2.9. Tous les résultats concernant $\mathbf{H}_{\mathbf{C}}(\mathbf{M})$ peuvent se voir dans $\mathbf{D M}_{\mathrm{gm}}(\mathbf{C})$ via le changement de base, puis en géométrie analytique, via [GAGA], comme chez Deligne. En particulier, le lemme 4.2.8.1. se réinterprète analytiquement en

$$
\mathbb{H}^{n}\left(\mathrm{R}^{\cdot} \operatorname{Hom}_{D_{a n}^{-}}\left(t_{a n}\left(\widetilde{\mathbf{M}}^{(\cdot)}\right), \boldsymbol{\Omega}_{a n}^{<q}\right)\right)=\mathbf{H}_{D R}^{n}(\mathbf{M}) / F^{q} \mathbf{H}_{D R}^{n}(\mathbf{M}) .
$$




\subsection{Réalisation de Deligne-Beilinson.}

Les résultats précédents permettent de généraliser aux motifs géométriques la définition de la cohomologie de Deligne-Beilinson. Pour les définitions des cohomologies de Deligne et DeligneBeilinson des schémas, nous nous référons à [Sc88] et [EV88].

Rappelons que pour un schéma $X$ lisse sur $\mathbf{C}$, le complexe de Deligne est défini pour tout entier $q$ comme le complexe de faisceaux en topologie analytique $D_{X}(q)$

$$
0 \rightarrow(2 i \pi)^{q} \mathbf{Z} \rightarrow \mathcal{O}_{X}^{h} \rightarrow \Omega_{X}^{1, h} \ldots \rightarrow \Omega_{X}^{q-1, h} \rightarrow 0
$$

où le terme $(2 i \pi)^{q} \mathbf{Z}=\mathbf{Z}(q)_{\text {an }}$ est en degré 0 . Ce complexe s'écrit

$$
D_{X}(q)=\text { cône }\left(\mathbf{Z}(q)_{a n} \rightarrow \Omega_{X}^{, h} / F^{q} \Omega_{X}^{, h}\right)[-1]
$$

et on pose $H_{D}(X, q)=H_{a n}^{\cdot}\left(X, D_{X}(q)\right)$.

Si le schéma $X$ est projectif, la théorie de Hodge et [GAGA] permettent d'inclure $H_{D}(X, q)$ dans une longue suite exacte

$$
\ldots \rightarrow H_{D}^{n}(X, \mathbf{Z}(q)) \rightarrow H^{n}(X(\mathbf{C}), \mathbf{Z}(q)) \rightarrow H_{D R}^{n}(X(\mathbf{C})) / F^{q} \rightarrow H_{D}^{n+1}(X, \mathbf{Z}(q)) \ldots
$$

où l'on voit que deux termes sur trois ont une interprétation motivique. Si le schéma n'est pas projectif, Beilinson [B84] a décrit le complexe en terme de complexe de De Rham à pôles logarithmiques pour définir une cohomologie de $X$ qui s'inscrit dans la même suite exacte longue. Comme dans le cas de la réalisation de Hodge on va résoudre un motif par son complexe des poids. Dans la catégorie dérivée des faisceaux analytiques sur $\operatorname{Smcor}(\mathbf{C})$, notons $\boldsymbol{\Omega}_{a n}^{<p}$ le complexe

$$
0 \rightarrow \mathcal{O}^{h} \rightarrow \boldsymbol{\Omega}^{1, h} \ldots \rightarrow \boldsymbol{\Omega}^{p-1, h} \rightarrow 0
$$

où l'anneau des fonctions holomorphes est en degré 0 et posons

$$
D(q)=\text { cône }\left(\mathbf{Z}(q)_{a n} \rightarrow \boldsymbol{\Omega}_{a n}^{<q}\right)[-1] .
$$

Soit $\mathbf{M}$ un motif géométrique effectif et $\mathbf{M}^{(\cdot)}$ un complexe des poids de $\mathbf{M}$. Par le théorème d'invariance d'homotopie, on identifie ces motifs de $\mathbf{D M}^{-}(\mathbf{C})$ à des complexes $\widetilde{\mathbf{M}}$ et $\widetilde{\mathbf{M}^{(\cdot)}}$ de $D^{-}\left(\operatorname{Shv}_{N i s}(\operatorname{Smcor}(\mathbf{C}))\right)$. Considérons le complexe

$$
D B_{q}(\mathbf{M})=\text { Tot } \mathrm{R}^{\cdot} \operatorname{Hom}_{D_{a n}^{-}}\left(t_{a n}\left(\widetilde{\mathbf{M}^{(\cdot)}}\right), D(q)\right) .
$$

Proposition 4.3.3. Pour tout motif géométrique effectif $\mathbf{M}$ le complexe $D B_{q}(\mathbf{M})$ est bien défini à homotopie près et sa cohomologie notée $H_{D}(\mathbf{M}, q)$ est isomorphe quand $\mathbf{M}$ est le motif d'un schéma lisse complexe $X$ à la cohomologie de Deligne-Beilinson de $X$.

Demonstration : le complexe $D B_{q}(\mathbf{M})$ est le cône de deux complexes bien définis à homotopie près : un calcule la réalisation de Betti (3.1.7) et le deuxième a été considéré en 4.2.9. Par 3.3.5, on a des isomorphismes

$$
\left.\mathbf{H}^{n}(\mathbf{M}(\mathbf{C}), \mathbf{Z}(q))=\operatorname{Hom}_{D_{a n}^{-}}\left(t_{a n}(\widetilde{\mathbf{M}}), \mathbf{Z}(q)_{a n}[n]\right)=\mathbb{H}^{n} \mathrm{R}^{\cdot} \operatorname{Hom}_{D_{a n}^{-}}\left(t_{a n}\left(\widetilde{\mathbf{M}}^{(\cdot)}\right), \mathbf{Z}(q)_{a n}\right)\right)
$$

et la suite exacte longue

$$
\ldots \rightarrow H^{n}\left(D B_{q}(\mathbf{M})\right) \rightarrow \mathbf{H}^{n}(\mathbf{M}(\mathbf{C}), \mathbf{Z}(q)) \rightarrow \mathbb{H}^{n} \mathrm{R}^{\cdot} \operatorname{Hom}_{D_{a n}^{-}}\left(t_{a n}\left(\widetilde{\mathbf{M}}^{(\cdot)}\right), \boldsymbol{\Omega}_{a n}^{<q}\right) \rightarrow H^{n+1}\left(D B_{q}(\mathbf{M})\right) \rightarrow \ldots
$$

qui, pour M motif d'un schéma lisse $X$, s'identifie à celle définissant la cohomologie de DeligneBeilinson.

Le produit sur le complexe de Deligne $D(q) \otimes D\left(q^{\prime}\right) \rightarrow D\left(q+q^{\prime}\right)$ est défini à partir du produit des différentielles et commute aux transferts :

$$
x \cup y=\left\{\begin{array}{ccc}
x . y & \text { si } & i=0 \\
x \wedge d y & \text { si } & i>0 \text { et } j=q^{\prime} \\
0 & \text { sinon } &
\end{array}\right.
$$


avec $x \in D(q)^{i}$ et $y \in D\left(q^{\prime}\right)^{j}$.

Le foncteur cohomologique $H_{D}$ est fonctoriel et induit un foncteur de la catégorie des motifs géométriques sur $\mathbf{C}$ vers la catégorie des anneaux gradués.

Si $\sigma: k \hookrightarrow \mathbf{C}$ est un plongement, on appelle réalisation de Deligne-Beilinson le foncteur cohomologique $H_{D, \sigma}=H_{D} \circ t_{\sigma}$ de $\mathbf{D M}_{\mathrm{gm}}(k)$ vers la catégorie des anneaux gradués. Si le plongement $\sigma$ est réel, on montre comme en (3.2) que la réalisation $H_{D, \sigma}(\mathbf{M})$ est muni d'une action de la conjugaison complexe $F_{\infty}$. On appelle réalisation de Deligne-Beilinson réelle le foncteur des invariants par $F_{\infty}$ de $H_{D, \sigma}$.

Remarque : dans la catégorie des complexes motiviques sur $\mathbf{C}$, nous avons défini en (2.1.5) des classes de Chern $\mathbf{Z}(q) \rightarrow \boldsymbol{\Omega}$ qui se factorisent à travers $\mathbf{Z}(q) \rightarrow_{\tau \leq q} \boldsymbol{\Omega}$; la projection $\mathbf{Z}(q) \rightarrow \boldsymbol{\Omega}^{<q}$ est triviale si l'on reste dans une catégorie de faisceaux algébriques, d'où la nécessité de passer par les réalisations analytiques. En effet, pour $q=1$, il n'existe pas de morphisme algébrique non trivial entre le groupe multiplicatif et le groupe additif. Pour définir une réalisation à la Deligne-Beilinson, il faut pouvoir construire une application logarithme.

\subsection{Comparaison avec les constructions de Huber}

Annette Huber construit ses réalisations en étendant à la catégorie $\mathbf{D M}_{\mathrm{gm}}(k)$ des motifs géométriques les foncteurs additifs qu'elle a définis en [H95]

$$
\tilde{R}: \operatorname{Sm}(k) \rightarrow C^{+}(\mathcal{A})
$$

de la catégorie des schémas lisses sur $k$ dans la catégorie des complexes bornés inférieurement d'une catégorie abélienne Q-linéaire $\mathcal{A}$ ( $\mathrm{cf}$ [H00], [H04] (theorem B.2.2)). Il est clair que, rationnellement, nos réalisations coïncident avec les siennes pour les motifs des variétés lisses et il reste à vérifier que nos constructions s'étendent de la même façon que la sienne. Comme elle le précise, il est important que les foncteurs $\tilde{R}$ soient à valeurs dans une catégorie de complexes et non dans une catégorie homotopique ou dérivée. Elle-même a construit, pour tout schéma lisse, des complexes [H95] $\tilde{R}_{\text {sing }}(X)$ et $\tilde{R}_{d R}(X)$.

Fixons-nous des résolutions injectives respectives $J_{D R}^{\cdot}$ du complexe de De Rham $\boldsymbol{\Omega}$ et définissons, pour un plongement $\sigma \hookrightarrow \mathbf{C}$ fixé, les foncteurs

$$
\begin{array}{cccc}
R_{D R}: \operatorname{Sm}_{(k)} & \rightarrow & \mathbf{C}^{+}\left(\mathcal{F}_{k}\right) \\
X & \mapsto \operatorname{Hom}_{C\left(\operatorname{Shv}_{N i s}(\operatorname{Smcor} k)\right)}\left(C_{*}\left(\mathbf{Z}_{t r}(X)\right), J_{D R}\right)
\end{array}
$$

où $\mathcal{F}_{k}$ est la catégorie des $k$-espaces vectoriels filtrés,

$$
\begin{array}{rlrl}
R_{B}: \operatorname{Sm} k & \rightarrow & C^{+}\left(\mathcal{E}_{\mathbf{Q}}\right) \\
X & \mapsto \operatorname{Hom}_{C(\mathcal{A} b)}\left(\operatorname{Map}\left(\Delta_{\text {top }}, \coprod_{d \geq 0} S^{d} X(\mathbf{C})\right)^{+}, \mathbf{Q}\right),
\end{array}
$$

où $\mathcal{E}_{\mathbf{Q}}$ est la catégorie des $\mathbf{Q}$-espaces vectoriels.

Pour tout schéma lisse $X$ le dual du morphisme de Dold-Thom fournit un quasi-isomorphisme

$$
u_{B}(X): \tilde{R}_{\text {sing }}(X) \simeq R_{B}(X)
$$

le complexe $\tilde{R}_{d R}(X)$ construit à partir des différentielles à pôles logarithmiques est une résolution du motif de De Rham et induit un quasi-isomorphisme

$$
R_{D R}(X) \simeq \tilde{R}_{d R}(X)
$$

compatible aux filtrations par les poids (2.2) et de Hodge (2.3).

L'essence des constructions de Huber $([\mathrm{H} 00](2,3))$ est d'étendre les foncteurs $\tilde{R}$ de $\operatorname{Sm} k$ à la catégorie Smcor $k$, la proposition (loc. cit. Prop.2.1.2) assurant qu'une fois choisie une règle de signes dans les multicomplexes, un foncteur de Smcor $k$ dans $C^{+}(\mathcal{A})$ vérifiant les bonnes propriétés s'étend naturellement en un foncteur $\mathbf{D M}_{\mathrm{gm}}(k) \rightarrow D^{+}(\mathcal{A})$.

Par construction, nos foncteurs $R$ s'étendent en des foncteurs sur $\operatorname{Smcor}(k)$. Il nous faut montrer que ces derniers coïncident avec les foncteurs étendus par Huber ([H00], theorem 2.1.6 et [H04] B.2.2), ce qui se ramène à vérifier que nous avons défini les mêmes transferts. 
Lemme 4.4.1. Pour toute correspondance $\alpha$ entre les schémas lisses $X$ et $Y$, on a un diagramme commutatif

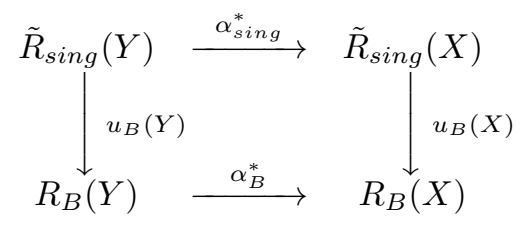

Demonstration : la construction des transferts sur $\tilde{R}_{\text {sing }}$ est explicitée dans la démonstration des théorèmes 2.1.3 et 2.1.6 de [H95]. Celle des transferts sur $R_{B}$ est, avant application du foncteur de réalisation topologique, issue de l'article [SV96] de Suslin et Voevodsky. Dans les deux cas on se ramène à une correspondance élémentaire de support qu'on peut supposer normal, et même un recouvrement génériquement galoisien chez Huber ou pseudo-galoisien chez Suslin et Voevodsky (les deux notions sont équivalentes en caractéristique 0). La même trace permet alors de définir les transferts qui coïncident.

La démonstration se transpose au cas des réalisations de De Rham et de Hodge puisque les transferts ([LW09]), puis les filtrations, ont été construits au niveau des complexes. La naturalité des isomorphismes de comparaison permet de conclure

Proposition 4.4.2. Nos foncteurs de réalisations $\mathbf{H}_{D R}$ et $\mathbf{H}_{\sigma}$ restreints à la catégorie $\mathbf{D} \mathbf{M}_{\mathrm{gm}}(k)$ des motifs géométriques coïncident respectivement avec les composantes de Rham et singulière du foncteur de réalisation mixte de Huber [HOO].

Par ailleurs, comme l'a précisé Bondarko ([Bo09], 7.4), il n'existe qu'une seule filtration par le poids sur une réalisation de $\mathbf{D M}_{\mathrm{gm}}$ vers une catégorie à coefficients rationnels. Nos filtrations par le poids coïncident donc avec celles de [H00], de même que les réalisations de Hodge.

\section{Bibliographie}

[SGA4] Artin, M. - Grothendieck, A. - Verdier, J. L. — Théorie des topos et cohomologie étale des schémas. Séminaire de Géométrie Algébrique du Bois-Marie 1963-1964 (SGA 4) Tome 1 : Théorie des topos, Lecture Notes in Mathematics 269 Springer-Verlag, Berlin, (1972).

[A10] Ayoub, Joseph. - Note sur les opérations de Grothendieck et la réalisation de Betti, J. Inst. Math. Jussieu, 9, (2010), no 2, 225-263.

[B84] Beıllinson, A. A.. - Higher regulators and values of $L$-functions, in Current problems in mathematics, Vol. 24, Itogi Nauki i Tekhniki, 9 , Akad. Nauk SSSR Vsesoyuz. Inst. Nauchn. i Tekhn. Inform., Moscow, (1984), 181-238.

[BV08] Beŭlinson, Alexander - Vologodsky, Vadim. - A DG guide to Voevodsky's motives, Geom. Funct. Anal., 17, (2008), no 6, 1709-1787.

[Bo09] Bondarko, Mikhael V. — Differential graded motives : weight complex, weight filtrations and spectral sequences for realizations; Voevodsky versus Hanamura , J. Inst. Math. Jussieu , 8, (2009), no 1, 39-97.

[Bo10] Bondarko, Mikhael V. — Weight structures, weight filtrations, weight spectral sequences, and weight complexes (for motives and spectra), J. K-Theory, 6, (2010), no 3, 387-504.

[CE] Cartan, Henri - Eilenberg, Samuel. - Homological algebra, Princeton Landmarks in Mathematics 6 Princeton University Press, Princeton, NJ, (1999).

[CD09] Cisinski, Denis-Charles - Déglise, Frédéric. — Local and stable homological algebra in Grothendieck abelian categories, Homology, Homotopy and Applications, 11, (2009), no 1, 219260 .

[D71] Deligne, Pierre. - Théorie de Hodge II, Inst. Hautes études Sci. Publ. Math., 40, (1971), no $1,5-57$. 
[D74] Deligne, Pierre. - Théorie de Hodge III, Inst. Hautes études Sci. Publ. Math., 44, (1974), no $1,5-77$.

[EV88] Esnault, Hélène and Viehweg, Eckart. — Deligne-Beŭlinson cohomology, in Beŭlinson's conjectures on special values of $L$-functions, Perspect. Math., 4 , Kluwer Acad. Publ., Boston, MA, (1988), 43-91.

[FV] Friedlander, Eric M. - Voevodsky, Vladimir. - Bivariant cycle cohomology, in Cycles, transfers, and motivic homology theories, Ann. of Math. Stud., 143, Princeton Univ. Press, Princeton, NJ, (2000), 138-187.

[Go58] Godement, Roger. - Topologie algébrique et théorie des faisceaux, Actualités scientifiques et industrielles 1252. Publications de l'Institut de Mathématique de l'Université de Strasbourg XIII Hermann, Paris, (1858).

[EGA IV] Grothendieck, A.. - Eléments de géométrie algébrique. IV. Étude locale des schémas et des morphismes de schémas IV, Institut des Hautes Études Scientifiques. Publications Mathématiques, XIII, (1967), no 32, 361.

[H95] Huber, Annette. - Mixed motives and their realization in derived categories, Lecture Notes in Mathematics 1604 Springer-Verlag, Berlin, (1995).

[H00] Huber, Annette. - Realization of Voevodsky's motives, Journal of Algebraic Geometry, 9, (2000), no 4, 755-799.

[H04] Huber, A.. - Corrigendum to : "Realization of Voevodsky's motives" [J. Algebraic Geom. 9 (2000), no. 4, 755-799], Journal of Algebraic Geometry, 13, (2004), no 1, 195-207.

[J90] Jannsen, Uwe. - Mixed motives and algebraic K-theory, Lecture Notes in Mathematics 1400 Springer-Verlag, Berlin, (1990).

[K73] Knighten, Carol M.. - Differentials on quotients of algebraic varieties, Transactions of the American Mathematical Society, 177, (1973), no 1, 65-89.

[L08] Lecomte, Florence. - Réalisation de Betti des motifs de Voevodsky, Comptes rendus Mathematique, 346, (2008), no 1, 1083-1086.

[LW09] Lecomte, Florence - Wach, Nathalie. - Le complexe motivique de De Rham, Manuscripta Math., 129, (2009), no 1, 75-90.

[Ma68] Manin, Ju. I. - Correspondences, motifs and monoidal transformations. (Russe), Izv. Akad. Nauk SSSR Ser. Mat. , 32, (1968), no 1, 1223-1244.

[MVW] Mazza, Carlo - Voevodsky, Vladimir - Weibel, Charles. - Lecture notes on motivic cohomology, Clay Mathematics Monographs 2 American Mathematical Society, Providence, RI, (2006).

[N89] Nisnevich, Ye. A. - The completely decomposed topology on schemes and associated descent spectral sequences in algebraic $K$-theory, in Algebraic $K$-theory : connections with geometry and topology (Lake Louise, AB, 1987), NATO Adv. Sci. Inst. Ser. C Math. Phys. Sci., 279 , Kluwer Acad. Publ., Dordrecht, (1989), 241-342.

[Sc88] Schneider, Peter. - Introduction to the Beulinson conjectures, in Beullinson's conjectures on special values of $L$-functions, Perspect. Math., 4, Kluwer Acad. Publ., Boston, MA, (1988), 1-35. [GAGA] Serre, Jean-Pierre. - Géométrie algébrique et géométrie analytique, Université de Grenoble. Annales de l'Institut Fourier, 6, (1955-1956), no 1, 1-42.

[SV96] Suslin, Andrei - Voevodsky, Vladimir. - Singular homology of abstract algebraic varieties, Inventiones Mathematicae, 123, (1996), no 1, 61-94.

[Ver77] Verdier, Jean-Louis. - Catégories dérivées, état 0, in Cohomologie étale, Séminaire de

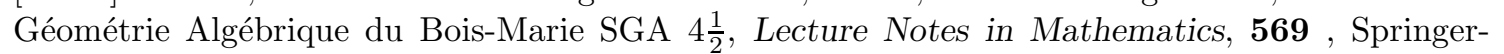
Verlag, Berlin, (1977), 262-311.

[Ver96] Verdier, Jean-Louis. - Des catégories dérivées des catégories abéliennes, Astérisque, 569, (1996), no 239, xii+253 pp. (1997).

[V-TCM] Voevodsky, Vladimir. - Triangulated categories of motives over a field, in Cycles, transfers, and motivic homology theories, Ann. of Math. Stud., 143 , Princeton Univ. Press, Princeton, NJ, (2000), 188-238. 